\title{
Journal homepage:
}

http://iiimct.journals.ekb.eg/

Online ISSN: $2682-2881$ Print ISSN: 2682-2105

\section{Original Research Article}

\section{Advanced knowledge services provided by national libraries in a knowledge society}

\section{Dr. Dina Adel Elsayed*.}

Department of Information Science, Electronic Archiving. Faculty of Arts,BeniSuef University, Egypt

\section{ABSTRACT}

This study aims to: Identify the forms of advanced knowledge services provided in national libraries, the emergence of which was associated with the emergence of the contemporary digital knowledge society, which are those services that are based on sharing and sharing knowledge to generate new knowledge and achieve the greatest possible benefit in order to build and develop a knowledge society, and the study followed the approach Descriptive and analytical as the most appropriate approach to achieve the objectives of the study, and the study reached a set of results, the most important of which are: Advanced knowledge services is a more advanced term than the term traditional information services, and it is also one of the new concepts that combines knowledge management, knowledge organization and knowledge markets Knowledge Markets, and among the forms of these knowledge services: (the library's website, the online catalog available on the direct line, the digital

\section{ARTICLE INFO}

Article history:

Received 2020-10-06

Accepted 2020-12-24

\section{Keywords:}

Knowledge Services: Sharing Knowledge: National Library

\footnotetext{
*Corresponding author:Email: dinaadelhussein@gmail.com
} 
reference service, the document supply service, the digital desktop media service, the electronic scientific publishing service, the electronic scientific translation service, the search and retrieval service with rules Scientific data, and the study came out with a set of recommendations, namely: The work system in contemporary national libraries should be developed to keep pace with the requirements of the knowledge society and the multiple needs of users by providing a set of knowledge services that depend on second generation web applications, web2.0, and smart phones applications

\section{ABSTRACT}

تهدف هذه الدراسة إلى: التعرف على أثكال الخدمات

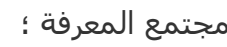

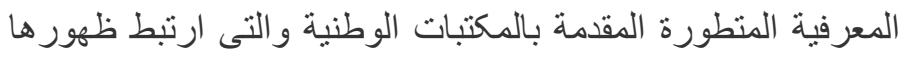

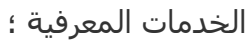

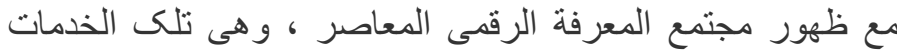

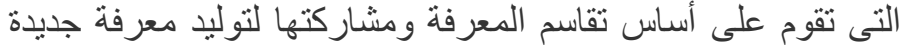
وتحقيق أكبر فائدة ممكنة بهدف بناء وتتمية مجتمع المعرفة ، و اتبعت التحتي مشاركة المعرفة ؛ - ات

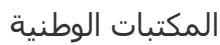

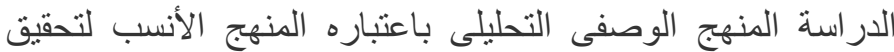
أهداف الدراسة ، وتوصلت الدراسة إلى مجمو عة من النتائج أهمها :

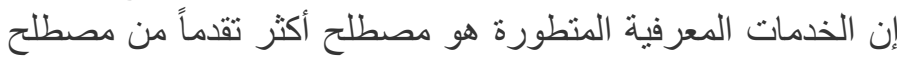
خدمات المعلومات التقليدية ، كما أنها إحدى المفاهيم الجديدة التى التى

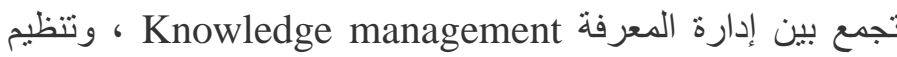

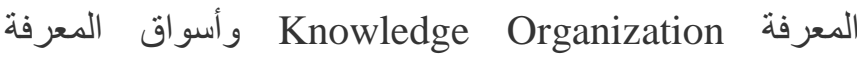
Knowledge Markets

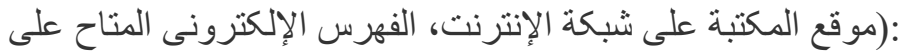

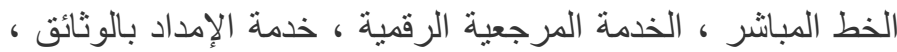

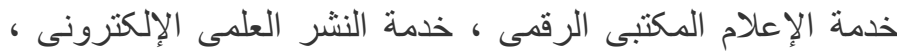

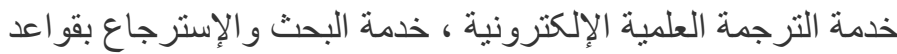

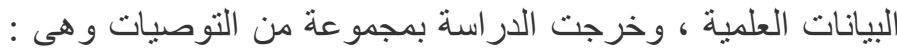

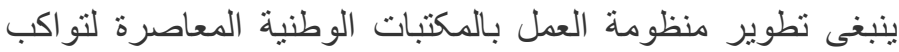

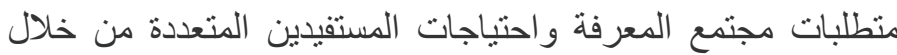

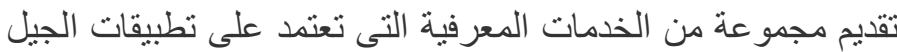

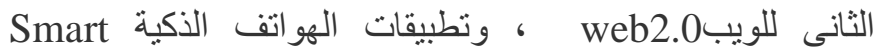
.Phones. 


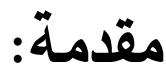

يبدو واضحاً أن العالم الآن في حالة تغير وأنه يمر بمرحلة جديدة في مسيرة تطوره ، فقد ظل لفترة طويلة من الزمن يدور في فلك المجتمع الزراعي القائم على المواد الأولية

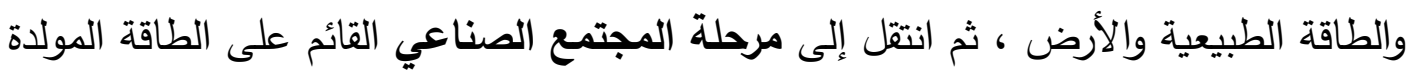

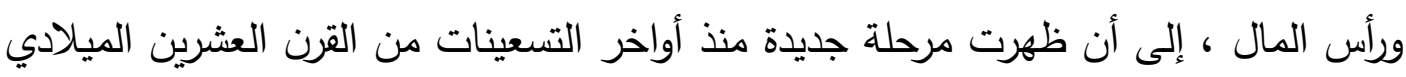
وهي مرحلة مجتمع المعلومات والذي عرفته القمة العالمية للمعلومات التي انعقدت بجنيف في ديسمبر 2003 على أنه " جميع الأنثطة والممارسات المرتبطة بالمعلومات إنتاجا ونشرا وتنظيما واستثمارا، والذي يعتمد في تطوره ونموه بصورة رئيسية على الحاسبات الآلية

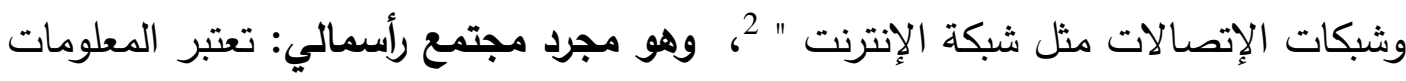

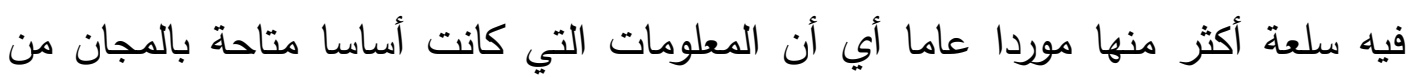
المكتبات العامة، والوثائق الحكومية أصبحت أكثر تكلفة عند الحصول عليها خصوصا بعد القيام بتخزينها في النظم المعتمدة على الحاسب الآلي، وهذه النظم مملوكة للقطاع الخاص، ويتم التعامل معها على أساس تجاري من أجل الربح. ولقد دار نقاش وتساؤلات في السنوات الأخيرة من القرن العشرين حول أن مرحلة

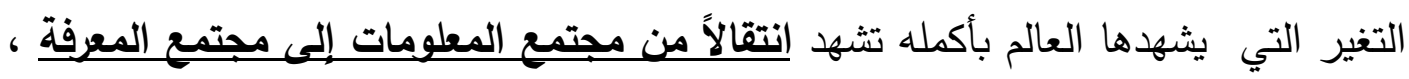

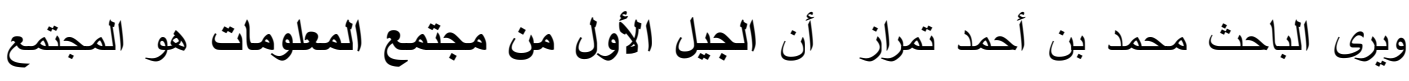

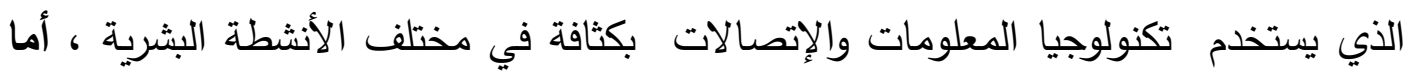
الجيل الثاني جيل مجتمع المعرفة فهو يتعدى المستوى التكنولوجي ليهدف إلى تثييد مجتمع

2 UNESCO.Towards Information Societies .paris. UNESCO Publishing .2005.p 27. 
مبدع ومبتكر يعتمد على قدر أكبر من الخبرة والممارسة والإبتكار لاى العنصر البشري أي أن جيل مجتمع المعرفة يهدف إلى المزج بين العوامل التكنولوجية والعوامل البشرية33. إن مجتمع المعرفة يشكل ظاهرة تستحق الدراسة بشكل وافي ومفصل يعرض لهذه القضية الهامة في مجتمعنا الرقمي المعاصر ، فمجتمع المعرفة هو ذلك المجتمع الذي يقوم على استغلال المعرفة كأهم مورد لتنمية جميع القطاعات الإقتصادية والنمو الإجتماعي بصفة عامة ، ومن ثم فإنه يتجاوز النفاذ إلى المعلومات وإتاحتها للجميع إلى تحويلها إلى موارد ملموسة من المعارف النظرية والتطبيقية والتكنولوجية والتنظيمية التي تسهم بصورة مباشرة في التنمية المستدامة للمجتمع - (ل)

وتسهم المكتبات بصفة عامة والمكتبات الوطنية بصفة خاصة في دعم وإرساء أسس مجتمع المعرفة ، وهناك صلة قوية بين المكتبات الوطنية وعصر مجتمع المعرفة ، حيث يتضح أهمية الدور الذي يمكن أن تقوم به المكتبات الوطنية في بناء مجتمع المعرفة من خلال حفظ وتتظيم الإنتاج الفكري الوطني وتوثيقه والتعريف به ونشره ، وإنطلاقاً من أهمية المكتبة الوطنية كمركز ثقافي معلوماتي يعكس تراث الأمة وتطورها العلمي والأدبي والثقافي وإسهاماً منها في نشر الثقافة العلمية التي أصبحت من ضروريات عصر مجتمع المعرفة . وتعد الخدمات المعرفية ثرة المكتبة ومخرجاتها Output التي تقوم على تقاسم المعرفة ومشاركتها لتوليد معرفة جديدة وتحقيق أكبر فائدة ممكنة بهدف بناء وتتمية مجتمع المعرفة ، كما أن التطبيقات المعرفية الجديدة في القرن الحادي والعشرين غيرت طريقة تقديم الخدمات في المكتبات حتى أننا بدأنا نسع في وقتتا الحالي مصطلح جديد إرتبط ظهوره Knowledge أيضاً بظهور مصطلح مجتمع المعرفة وهو مصطلح الخدمات المعرفية

محمد بن احمد نمراز ـ حول بعض مؤشرات مجتمع المعلومات .ـ- المجلة العربية للعلوم والمعلومات ، ع 53 . 32005

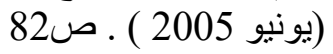


Information كمرحلة أكثر تطوراً وتقدماً من مصطلح خدمات المعلومات Services . Services

وقد استخدم قاموس ODLIS عبارة الخدمات المتاحة على الخط المباشر On- Line Services كمرادف للتعبير عن الخدمات المعرفية المكتبية الإكترونية حيث يعرفها بأنها " قطاع من الخدمات العامة التي تقدمها المكتبة تعني بإنتقاء وإتاحة إمكانية النفاذ إلى المصادر الإكترونية مثل الفهارس الإكترونية وقواعد البيانات الببليوجرافية والتي عادة ما تتطلب وساطة للبحث عنها والإستفادة منها يقوم بها أخصائي المكتبات المكلف بالخدمات المتاحة على الخط المباشر " 4

ومما لا شك فيه فإن المكتبة الوطنية لا تستطيع توفير الخدمة المعرفية دون أن يتوافر لها مجموعة من المقومات والمتطلبات التي يمكن إيجازها فيما يلي: 1. توفير مجموعة متكاملة ومتوازنة من مصادر المعلومات التقليدية والرقمية وذلك لتلبية الإحتياجات المختلفة والمتغيرة لمجتمع المستفيدين • 2. إعداد وتجهيز وتتظيم هذه المصادر ، وتوفير نظم الإسترجاع الملائمة من فهارس وكثافات ومستخلصات وذلك لتوفير وقت وجها المستفيدين في عمليات البحث والتحليل . 3. توفير الأخصائيين المؤهلين القادرين على تقديم الخدمات المعرفية المتطورة على - النحو الملائم للمستفيدين 4. الإمكانيات التكنولوجية لتقديم هذه الخدمات . 5. تقديم الخدمات في المكان المناسب لوصول المستقيدين إليه .

${ }^{4}$ ODLIS.(n.d).ABC-CLIO. Electronic Services .- Available at : http://www.abcclio.com/ODLIS/odlis_o.aspx\#onlineservices cited in 2/5/2017 
6. توافر الإمكانيات المادية لتغطية النفقات لتنمية وبناء مصادر المعلومات ، وشراء

$$
\text { الأجهزة والبرمجيات وصيانتها }
$$

7. موقع المكتبة على شبكة الإنترنت ومدى سهولة إستخدامه .

\section{ثانياً : مشيكلة الدراسة}

تتمثل مشكلة الاراسة الحالية في الإجابة على التساؤل الرئيس التالي :

ما أشكال وأنواع الخدمات المعرفية المنوط بالمكتبة الوطنية تقديمها في عصر

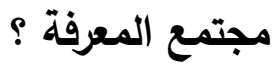

ويتفرع من التساؤل الرئيس التساؤلات الفرعية التالية :

1- ما المقصود بمصطلح الخدمات المعرفية المتطورة ؟

2- ما أثكال وأنواع الخدمات المعرفية الجديدة في ظل مجتمع المعرفة ؟

3- ما المقومات اللازمة للمكتبات الوطنية لتقديم مجموعة من الخدمات المعرفية

المتطورة لجمهور المستقيدين منها ؟

4- ما الدور الأساسي الذي يقع على عاتق أخصائيين المعرفة العاملين بالمكتبة

الوطنية من أجل تقديم مجموعة من الخدمات المعرفية لجمهورالمستقيدين ؟

\section{ثُالثَاً: أهداف الدراسة :}

1- تعريف مفهوم الخدمات المعرفية المتطورة المقدمة بالمكتبات في عصر مجتمع

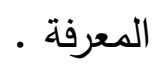

2- تحديد أشكال وأنواع الخدمات المعرفية المتطورة المنوط بالمكتبات تقديمها في ظل

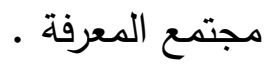


3- دراسة دور أخصائيين المعرفة العاملين بالمكتبات الوطنية في تقديم مجموعة من

الخدمات المعرفية للمستفيدين من خدمات المكتبة الوطنية .

\section{إبعاً: منهج الدراسة}

- المنهج الوصفي التحليلي : من أجل رسم وتحديد الإطار النظري لموضوع الخدمات المعرفية المتطورة المقدمة بالمكتبات الوطنية ، وذلك بإستقراء الإنتاج الفكري ذي الصلة المباشرة وغير المباشرة بموضوع الخدمات المعرفية .

\section{خامساً : مصطلحات الدراسة :}

\section{:Knowledge Concept : المعرفة}

"المعلومات التي تُقيم وتتظم في العقل لإستخدامها فيما بعد لتحقيق أهداف معينة ، والمعرفة هي أحد العناصر الأساسية ضمن سلسلة متكاملة تبدأ بالبيانات ثم المعلومات ثم المعرفة وأخيراً الحكمة ، والمعرفة هي خلاصة تجميع وتقويم وتنظيم المعلومات بثكل مفيد في ضوء الخبرة حول موضوع معين أو هي مزيج من الخبرات والمهارات المتراكمة لدى العاملين بالمنظمة ، وهناك نوعان للمعرفة وهما المعرفة الضمنية والمعرفة الصريحة "5

\section{محتمع المعرفة : Knowledge Society :}

"المجتمع الذي يقوم على نشر المعرفة وإنتاجها وتوظيفها بكفاءة في جميع مجالات

النشاط المجتمي : الإقتصاد، المجتمع المدني، السياسة، الحياة الخاصة، وصولا لترقية الحالة الإنسانية بإطراد أي إقامة التنمية الإنسانية الثاملة، وفي هذا المجتمع تلعب المعرفة دورا حاسماً وبارزاً في تشكيل البنية المجتمية الأساسية وأدائها في مجالات الإقتصاد

${ }^{5}$ Koenig, Micheal . International Encyclopedia of Information and Library Science .- London.Rout- Ledge, 2003. P 351 
والسياسة والمجتمع المدني، وفي حياة أعضاء هذه المجتمعات بحيث يتكثف المدخل المعرفي في الحياة اليومية لهج، وفي مجال العمل على وجه الخصوص يزداد عدد العاملين في منظومة المعرفة، وترتفع نسبة وقت العمل المخصصة للنشاطات المعتمدة على كثافة

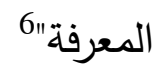

\section{:Knowledge Services الخدمات المعرفية}

" تلك الخدمات التي تقوم بها المكتبات من خلال إدارة المعرفة وتتظيمها وإتاحتها لتمكين المستفيدين من الوصول إلى المعرفة التي يحتاجون إليها بأسرع وقت وأقل جهد وأكثر فاعلية وذلك بإستخدام تكنولوجيا المعلومات والإتصالات في عصر مجتمع المعرفة ، والخدمات المعرفية الرقمية تتمثل في : المصادر الرقمية للمعلومات Resources Digital-، الخدمات المرجعية الرقمية Digital Reference Services ، موقع المكتبة على شبكة الإنترنت ، الفهرس المتاح على الخط المباشر OPAC ، خدمة الإمداد بالوثائق ، خدمة الإعلام المكتبي الرقمي ، خدمة الترجمة العلمية ، النشر العلمي الإلكتروني "7"

\section{Knowledge Directories : مديرين المعرفة}

" الأفراد القائمين على أمر إدارة المعرفة ، والمشرفين على تتفيذ عملياتها الست (تثخيص المعرفة وتحديد أهدافها - تكوين المعرفة واقتناؤها - تتظيم المعرفة واختزانها"8" توزيع المعرفة وبثها - تطبيق المعرفة واستخدامها )

65 UNESCO . UNESCO National Report about ( From Information Society Towards Knowledge Society . Paris . UNESCO, 2005. - Available at : https://unesdoc.unesco.org/ark:/48223/pf0000141843 ara cited in 1/8/2015

${ }^{7}$ ODLIS.(n.d).ABC-CLIO. Electronic Services .- Available at : http://www.abcclio.com/ODLIS/odlis_o.aspx\#onlineservices cited in 2/5/2017.

السيد السيد النشار ـ أساسيات إدارة المعرفة .ـ الإسكندرية : دار الثقافة العلمية ، 2012 ـ ص 215 . 


\section{سادساً: الخدمات المعرفية المتطورة : أشكالها وأنواعها}

\section{ومقوماتها :}

تعد الخدمات المعرفية ثرة الدكتبة ومخرجاتها Output التي تقوم على تقاسم

المعرفة ومشاركتها لتوليد معرفة جديدة وتحقيق أكبر فائدة مدكنة بهدف بناء وتنمية مجتمع المعرفة ، كما أن التطبيقات المعرفية الجديدة في القرن الحادي والعشرين غيرت طريقة تقديم الخدمات في الككتبات حتى أننا بدأنا نسمع في وقتتا الحالي مصطلح جديد إرتبط ظهوره

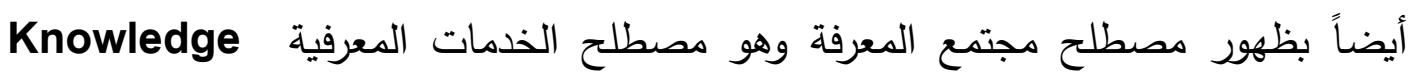

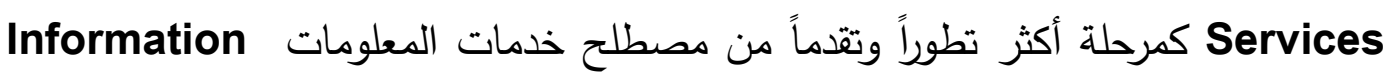

\section{. Services}

وقد استخلم قاموس ODLIS عبارة الخدمات المتاحة على الخط المباش1On- Line Services كمرادف للتعبير عن الخدمات المعرفية المكتبية الإكترونية حيث يعرفها بأنها " قطاع من الخدمات العامة التي تقدمها المكتبة تعني بإنتقاء وإتاحة إمكانية النفاذ إلى الدصادر الإلكترونية مثل الفهارس الإلكترونية وقواعد البيانات الببليوجرافية والتي عادة ما تتطلب وساطة للبحث عنها والإستفادة منها يقوم بها أخصائي البهائيه المكتبات المكلف بالخدمات المتاحة على الخط المباشر " 9

Knowledge فقد عرفت الذذمات المعرفية Wikipedia أما موسوعة Knowledge بأنها " إحدى الدفاهيم الجديدة التي تجمع بين إدارة الدعرفة Services وتنظيم المعرفة Management Knowledge Markets ، وهي عبارة عن البرامج التي تقدم مخرجات تنظيمية قائمة على

\footnotetext{
9 8ODLIS.(n.d).ABC-CLIO. Electronic Services .- Available at : http://www.abcclio.com/ODLIS/odlis_o.aspx\#onlineservices cited in 2/5/2017.
} 
محتوى مكونات الطيف المعرفي (البيانات Data - المعلومات Information- المعرفة ) ومن هذه الخدمات نذكر على سبيل المثال (تقديم المشورة Advice ) - الرد على التساؤلات Answers - التسهيلات Facilities ) بهدف تلبية إحتياجات

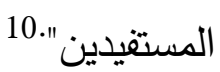

11 : Clair كما يلي العناصر المكونة للخدمات المعرفية فقد حددها الباحث 1) إدارة المعلومات Information Management : يقصد بها الحصول على المعلومات - تخزينها - تتظيمها -إسترجاعها - ومن ثم إستخدامها .

2) إدارة المعرفة Knowledge Management : ويقصد بها إدارة المعلومات الضمنية والصريحة بطرق تعزز إعادة إستخدامها وخلق معرفة جديدة . 3) الإستراتيجية Strategic في التدريب والتعليم :لتحقيق النجاح والتميز بالأداء في

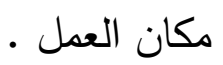

مما سبق نستخلص التعريف التالي لمصطلح الخدمات المعرفية وهي " تلك العمليات التي تقوم بها المكتبات من خلال إدارة المعرفة وتتظيمها وإتاحتها لتمكين المستفيدين من الوصول إلى المعرفة التي يحتاجون إليها بأسرع وقت وأقل جه وأكثر فاعلية وذلك بإستخدام تكنولوجيا المعلومات والإتصالات في عصر مجتمع المعرفة ، والخدمات المعرفية الرقمية تتمثل في : المصادر الإكترونية E-Resources ، الخدمات المرجعية الرقمية Digital Reference Services الإجتماعي Social Media ، والتسويق الإلكتروني لخدمات المكتبة Electronic Marketing ، وتوفير المساحات المادية ضمن مبنى المكتبة وذلك لعدة أغراض منها : $10 \quad 9 \quad$ Knowledge Services (n.d) .Available at : http://en.wikipedia.org/wiki/Knowledge services cited in 3/5/2017

${ }_{11}$ Clair,G. Knowledge Services :The EOS International Approach.-Available at :http://www.eosintl.com/wp-content/uploads/2011/12/EOS-knowledge services (2011) 
التعليم ، المحاضرات ، الندوات ، ورش العمل ، دورات محو الأمية المعلوماتية وغيرها من

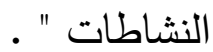

ومما لا شك فيه فإن المكتبة الوطنية لا تستطيع توفير الخدمة المعرفية دون أن

يتوافر لها مجموعة من المقومات والمتطلبات التي يمكن إيجازها فيما يلي : (2) 12 توفير مجموعة متكاملة ومتوازنة من مصادر المعلومات التقليدية والرقمية وذلك لتلبية الإحتياجات المختلفة والمتغيرة لمجتمع المستفيدين . إعداد وتجهيز وتنظيم هذه المصادر ، وتوفير نظم الإسترجاع الملائمة من فهارس وكثافات ومستخلصات وذلك لتوفير وقت وجهد المستقيدين في عمليات البحث والتحليل .

توفير الأخصائيين المؤهلين القادرين على تقديم الخدمات المعرفية المتطورة على النحو الملائم للمستقيدين الإمكانيات التكنولوجية لتقديم هذه الخدمات . تقديم الخدمات في المكان المناسب لوصول المستفيدين إليه . توافر الإمكانيات المادية لتغطية النفقات لتنمية وبناء مصادر المعلومات ، وشراء. الأجهزة والبرمجيات وصيانتها موقع المكتبة على شبكة الإنترنت ومدى سهولة إستخدامه.

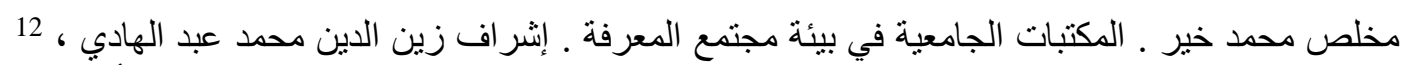

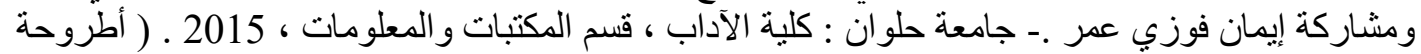

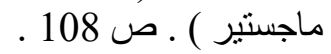




\section{أنواع وأشكال الخدمات المعرفية بالمكتبة الوطنية :}

\section{1- موقع المكتبة الوطنية على شبكة الإنترنت Web Site :}

أصبح من الضروري في عصر مجتمع المعرفة أن يكون للمكتبات الوطنية مواقع إلكترونية على شبكة الإنترنت حتى تستطيع تقديم خدماتها المعرفية (الفهرس المتاح للجمهور على الخط المباشر On-Line Public Access Catalog"OPAC ، الخدمة المرجعية الرقمية ، مجموعات المكتبة الرقمية ، التعليم عن بعد ، الأدلة الإرشادية ، البحث والإسترجاع في قواعد البيانات وغيرها من الخدمات المعرفية ، والموقع الإلكتروني للمكتبة الوطنية إنما هو مجموعة من المزايا والخدمات التي تجذب الباحث عن المعلومة أو المستفيد مما يساهم في تسهيل سبل الوصول إلى المعلومات وبناء وتتمية مجتمع المعرفة

\section{OPAC (ON-Line : الفهرس المتاح على الخط المباشر}

\section{يُعرف الفهرس المتاح على الخط المباشر : Public Access Catalog)}

بأنه: " قاعدة بيانات ببليوجرافية تتكون من تسجيلات ببليوجرافية تصف الكتب ومصادر المعلومات الأخرى وغالباً ما تتيح هذه الفهارس البحث بعدة خيارات أهمها: المؤلف، العنوان، الموضوع، الكلمات المفتاحية، كما تسمح للمستفيدين بطباعة وتحميل أو تصدير التسجيلات

$$
\text { البيليوجرافية عبر البريد الإكتروني ") } 13
$$

وهذا التعريف لايشمل الدور الجديد لهذا الفهرس الذي بدأت ملامحه وإمكاناته تتوافق مع إمكانيات الجيل الثاني للويب (Web 2.0 ) ، والذي أصبح فيه الفهرس يمثل بيئة تشابكية قابلة للعرض والكتابة وليس مجرد أداة للبحث والإسترجاع وأطلق عليه

يونس أحمد الشوابكة .إستخدام الفهارس العربية المتاحة للجمهور على الخط المباشر :فهرس مكتبة 13

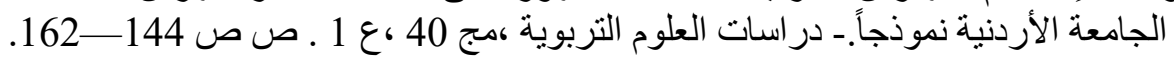


بموجب هذه الإمكانات إسم (S-OPAC) أي الفهرس الإجتماعي المتاح للجمهور على الخط المباشر

ويعرف أحمد المصري الفهارس الإجتماعية المتاحة للجمهور على الخط المباشر بأنها " فهارس إلكترونية متاحة للجمهور على الخط المباشر ويجري إستخدامها والتقاعل معها عبر واجهات إستخدام رسومية متاحة على شبكة الويب ، وتقوم على تتميتها وإدارة محتوياتها مكتبة أو أكثر ، ويتيح هذا الفهرس الوصول إلى قواعد البيانات الإككترونية ، والمستودعات الرقمية ، والأرشيفات مفتوحة المصدر )

\section{3- الخدمة المرجعية الرقمية :}

إن الخدمة المرجعية واحدة من أهم وأقدم الخدمات المقدمة في المكتبات ومراكز المعلومات ، ولم تكن الخدمة المرجعية بمعزل عن التأثر بالتطورات التكنولوجية التي طالت كافة أنشطة المكتبات ، فكما دخلت الميكنة إلى فهارس المكتبات وأُتيحت على الإنترنت تأثرت الخدمة المرجعية بالتطورات التكنولوجية الحديثة وظهر ما يُعرف بالخدمة المرجعية الرقمية وهي شكل جديد للخدمة المرجعية يُقدم عبر شبكة الإنترنت .

ALA ولقد عرف قسم خدمات المراجع والمستفيدين بجمعية المكتبات الأمريكية الخدمة المرجعية الرقمية بأنها " خدمة مرجعية تقدم إلكترونياً ، حيث يستخدم المستفيد الحاسب الآلي أو أي تقنية إنترنت للإتصال بأخصائي المراجع دون الحضور مادياً إلى المكتبة ، ويتم في الغالب إستخدام المصادر المتاحة على الخط المباشر لتقديم الخدمة ، وأصبحت المكتبات الوطنية تقدم خدماتها المرجعية في عصر مجتمع المعرفة بصورة أكثر Skype فعالية وسرعة وكفاءة ، حيث بدأت المكتبات إستخدام مكالمات الفيديو مثل برنامج

أحمد حسين بكر المصري ـ الفهارس الإجتماعية المتاحة على الخط المباشر :دراسة تحليلية للمتطلبات 14 الفنية و الوظيفية لتصميم نموذج عربي .- جامعة حلوان : كلية الآداب ، قسم المكتبات و المعلومات المبرة ، 2013 ـ (أطروحة دكتور اه) ، ص 16 ـ 2013 . 
وغيرها من التقنيات الحديثة ، ويطلق على الخدمة المرجعية الرقمية عدة تسميات باللغة الإنجليزية نذكر منها :

Electronic Reference, On-Line Reference, Ask A

15 Digital Reference, Life Reference, Librarian"

ولقد وضعت الباحثتان باوية بسيوني وسوسن ضليمي أسس ومتطلبات تقديم الخدمة المرجعية بالمكتبات الوطنية في الدراسة التي أعدتاها حول الخدمة المرجعية الرقمية بالمكتبات الوطنية فيما يلي : (2) 16 - البنية الأساسية -

- يجب أن يتوافر لأخصائي المراجع أماكن مناسبة وتوفير أجهزة حاسبات وبرامج لإدارة الخدمة ، بالإضافة إلى متخصصين في تكنولجيا الحاسبات والمعلومات للدعم الفني والصيانة.

-تلك التجهيزات المادية والبرمجيات المستخدمة لتقديم الخدمة المرجعية يجب ألا تبتعد عن ما هو متاح بالفعل لدى المستثيد . - أن تكون جميع الأجهزة والبرمجيات المستخدمة لتقديم الخدمة المرجعية حديثة وجيدة للحفاظ على كفاءة الخدمة وفعاليتها .

${ }^{15}$ RUSA. GuideLunes for Implementing and Main ting Virtual Reference Service (2014) .- Available at : http://dx.doi.org/10.5860/rusq.50nl.92 cited in 23/5/2017.

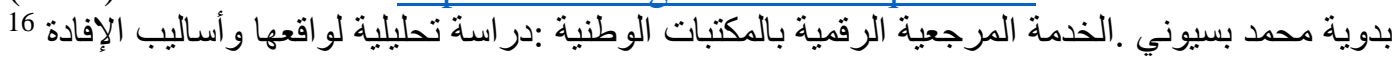

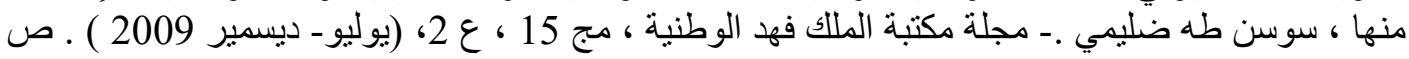
121 


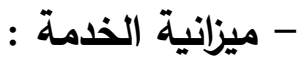

- ضرورة توفير التمويل المستمر للخدمة ، حتى وإن نشأت عن طريق منحة مالية مجانية تقدم لمرة واحدة أو عند قيام الخدمة كتجربة رائدة .

- يجب أن يتم تحديد ما إذا كانت الخدمة سوف تقدم للمستقيدين بشكل مجاني أم •بمقابل مادي

\section{- العاملون بالخدمة :}

- يجب أن يتم إختيار الأخصائيين للعمل في الخدمة المرجعية الرقمية على أسس علمية تعتمد على قدرتهم على الأداء ، كما يتم تدريب الأخصائيين المرجعيين على مهارات التعامل مع شبكة الإنترنت . - مع

إكساب العاملين لبعض المهارات البرمجية مثل تصميم صفحات الويب وإدارة قواعد

- ضبط الجودة (Quality Control) وتثمل المعايير الأساسية للأسئلة المرجعية - أنواع مصادر المعلومات المستخدمة - أشكال الإجابة على الأسئلة - الإجابة من المصادر المتاحة والإحالة إليها ، ويجب أن يتاح للأخصائيين الوقت والمصادر اللازمة للتعليم والتدريب المستمر لضمان خدمة فعالة .

\section{- تسويق الخدمة : - ت}

- من الضروري وضع خطة عامة لتسويق الخدمة المرجعية الرقمية وتنفيذ هذه الخطة ضمن جدول زمني محدد ، كما يجب تحديد الجمهور المستهدف من هذه الخدمة وان يكون التسويق مناسباً لهذا الجمهور . 
- تقييم الخدمة المرجعية الرقمية :

- من الضروري تحليل الخدمة المرجعية الرقمية بشكل منتظم ، ويتم الحصول على البيانات اللازمة للتقويم من الأخصائيين ومن المستفيدين لقياس فعالية الخدمة وكفاءتها ، ويجب أن يتم إستخدام نتائج التقييم في تحسين الخدمة ورفع مستوى تقديمها . مما سبق نجد أن الخدمة المرجعية الرقمية من أهم الخدمات المعرفية التي يجب أن تقوم بتقديمها المكتبات الوطنية لمواكبة عصر المعرفة الرقمي من خلال وضع إستراتيجية لتقديم هذه الخدمة تتضمن (خطوات الإجابة عن الأسئلة باللغة العربية والإنجليزية والإحتفاظ بالإجابات السابقة لإعادة إستخدامها و سياسة تقديم الخدمة المرجعية الرقمية بالمجان ، كذلك سياسة تحديد المدى الزمني المستغرق للرد على الإستفسارات ، سياسة تحديد عدد الأسئلة المسموح بها للمستفيد في المرة الواحدة ، وكذلك أيضاً تحديد الحد الأدني من بيانات المستفيد المقبولة مثل : الإسم - البربد الإكتروني (الإستفسار )

\section{4- مجموعات المكتبة الوطنية الرقمية : National}

\section{Library Collectio}

أصبحت التكنولوجيا واقعاً ملموساً في حياة الأفراد ، فأصبح المستقيد من المكتبة الوطنية يفضل شاشات الكمبيوتر للإطلاع على الكتب بدلاً من السير بين رفوف المكتبة لمعاينة ما يريد قراءته وتصفحه ، وإستجابة لهذا التحول النوعي في سلوكيات المستفيدين إختارت العديد من المكتبات الوطنية العالمية رقمنة بعض أو كل محتوياتها كمحاولة لمواكبة بيئة مجتمع المعرفة. 
وهناك عدة تجارب لمكتبات وطنية أجنبية مثل : مكتبة الكونجرس الأمريكية ، مكتبة كندا الوطنية - المكتبة الوطنية البريطانية ...إلخ ) ، وأيضاً هناك عدة محاولات عربية ناجحة لرقمنة مجموعات المكتبات الوطنية العربية مثل مشروع المحتوي الرقمي لمكتبة الملك فهد الوطنية السعودية - رقمنة مجموعات مكتبة دبي الوطنية الرقمية بدولة الإمارات العربية ..إلخ ) ، إذن خدمة إتاحة مجموعات المكتبة الوطنية بثكل رقمي على موقع المكتبة الوطنية على شبكة الإنترنت من أهم الخدمات التي يجب أن تسعى المكتبات الوطنية على تقديمها لجمهور المستقيدين منها ـ

\section{5ocument Delivery : خدمة الإمداد بالوثائق}

\section{Service(DDS)}

عرف قاموس ODLIS خدمة الإمداد بالوثائق بأنها " تقديم الوثائق المنشورة وغير المنشورة للمستفيدين في شكل مطبوع ، أو على أشكال مصغرة ، أو في شكل رقمي ، وهي خدمة تقدم عادة عند طلبها في مقابل رسوم ثابتة ، وفي معظم المكتبات يتم تقديم الخدمة من مكتب الإعارة التبادلية Interlibrary Loan ، ويطلب من المستفيدين عادة إستلام الوثائق الورقية من المكتبة ، أما الوثائق الإكترونية فيمكن إرسالها لهم عبر البريد الإكتروني:17

17 ODLIS.(n.d) .ABC-CLIO. Document Delivery Service .- Available at : http://www.abc-clio.com/odlis/searchODLIS.aspx cited in 25/6/2017 
وقد حددت الباحثة إيمان فتحي محمود في الدراسة الأكاديمية التي قامت بإعدادها حول خدمة الإمداد بالوثائق في المكتبات الوطنية الخطوات التالية لتقديم الغدمة بشكل رقمي للمستفيد من المكتبة الوطنية:18

1- المستفيد : وهو العنصر الأساسي الذي من أجله طورت خدمة الإمداد بالوثائق

إلكترونياً ، فتسعى المكتبة الوطنية الرقمية في عصر مجتمع المعرفة في دراسة إحتياجات المستقيدين والعمل على إددادهم بالوثائق العلمية الذين يريدون الإطلاع عليها في شكل رقمي.

2- البحث عن الوثائق ومصادر المعلومات الإكترونية : يعد البحث عن الوثائق هو أول خطوات الإمداد بالوثائق إلكترونياً إذ يجب على المستفيد أن يحدد الوثائق المطلوبة والتي من الأفضل أن تلبي إحتياجاته بثكل مباشر مما يساهم في توفير الوقت والجهد اللازم للوصول إلى تلك الوثائق ، وهنا ييرز دور أخصائي المعلومات المؤهل والمدرب للتعامل بكفاءة ومهارة مع برامج وأجهزة الإتصالات وتوظيفها في خدمة الإمداد بالوثائق المقدمة . 3- طلب المستفيد للوثائق : ويتم ذلك بالتحدث مع أخصائي الإمداد بالوثائق أو القيام بتعبئة نموذج الإمداد بالوثائق سواء كان في شكل ورقي أو إلكتروني ، وقد يستخدم الهاتف أو البريد العادي أو البريد الإكتروني أو شبكة الإنترنت لطلب الوثائق من المكتبة . 4- التعرف على مصادر الإمداد بالوثائق : في هذه المرحلة يتم إختيار الجهة المناسبة للحصول منها على الوثيقة من أجل هذا لابد من معرفة مكان كل وثيقة على حدة ليتم طلبها من المكتبات الأخرى أو من الناشربين المحتمل توافر الوثيقة لديهم وتتتوع مصادر

إيمان محمد فتحي محمود ـ خدمة الإمداد بالوثائق في المكتبات الوطنية :دراسة تحليلية مع وضع تصور 18

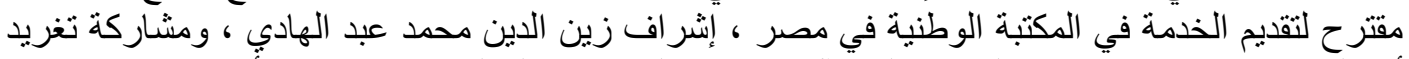

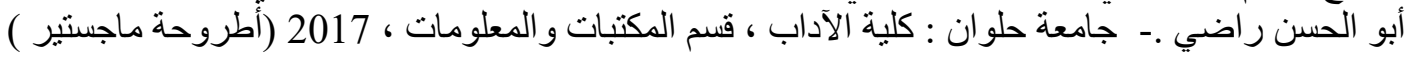

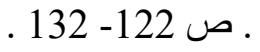


الإمداد بالوثائق بحيث تشمل : (المكتبات ، إتحادات المكتبات ، الجمعيات ، متعهدي الإشتراكات ، الناشريين ، المؤلفين ) ·

5- التجهيزات والمعدات : بمرور الوقت أصبحت المكتبات ومؤسسات المعلومات تسعى إلى إستخدام التكنولوجيا المتطورة في تقديم خدماتها ، ولأن خدمة الإمداد بالوثائق إلكترونياً مثل باقي الخدمات التي تقدمها المكتبات الوطنية فإن الإهتمام بمستوى التجهيزات والمعدات يحسن من مستوى الأداء عند تقديمها حيث تحتاج الخدمة إلى العديد من الحاسبات الآلية ، الطابعات ، الماسحات الضوئية ، الإتصال بشبكة الإنترنت فائق السرعة وذلك لتغطية كافة أنحاء العالم •

6- معالجة الطلبات من قبل المورد : عندما تصل الطلبات إلى المورد - سواء كان مكتبة أو ناشر تجاري - لابد من معالجة الطلب ، حيث تضاهي البيانات البيليوجرافية للوثائق المطلوبة بفهرس المكتبة الوطنية أو خدمة الإمداد التجارية ، ثم تُسترجع الوثائق وتُجمع وتُرسل ، وقد ساعدت التكنولوجيا الحديثة في هذه المرحلة ، حيث ظهرت نظم المضاهاة الإلكترونية لمضاهاة المقتتيات بالطلبات .

7- ضبط الجودة : يتعين على مقدم خدمة الإمداد بالوثائق إلكترونياً أن يهتم بجودة

الخدمة التي يقدمها ، فضبط الجودة يعد من الأمور المهمة ، سواء أكانت الخدمة تقدم بمقابل أو بدون مقابل ، وتبدأ هذه العملية مع الخطوات الأولى لخدمة الإمداد بالوثائق إذ أنه يجب التأكد من البيانات البيليوجرافية الصحيحة للوثائق المطلوبة مما يقلل من الوقت والتكلفة الذين تستهلكهما البيانات غير الصحيحة .

8- إيصال الوثائق والإمداد بها إلكترونيا: أصبحت تُستخدم للإمداد بالوثائق بشكل إلكتروني شبكة الإنترنت عن طريق إرسال نسخة PDF للوثيقة المطلوبة وما على المستفيد إلا أن يضغط على الزر لفتح الوثيقة وهو في مكانه وأيضاً دفع قيمة الإمداد بالوثائق بشكل إلكتروني عن طريق كروت الإعتمان ، أو بالإيداع في الحساب المخصص للخدمة في البنك . 


\section{6igital Mass Media : خدمة الإعلام المكتبي الرقمي}

يعد الإعلام من أهم الدعائم والركائز الأساسية لقيام دولة قوية ، وقد أثبت الإعلام

ذلك في الآونة الأخيرة حيث ظهر دوره الفعال في تتاقل وإنتشار المعلومات وقد أثرت التطورات التكنولوجية الحديثة بشكل كبير على الإعلام بشكل عام مما أدى إلى ظهور مصطلح الإعلام الرقمي الذي أصبح أكثر إنتشاراً بين طبقات المجتمع المختلفة .

وإن وسائل الإعلام الرقمية أصبحت عامل أساسي لابد وأن تعتمد عليه المكتبات الوطنية للتسويق لأقسامها وخدماتها ومجموعاتها ، وإستخدام المكتبة الوطنية لتلك القنوات الرقمية يساعد على وصولها إلى قطاع واسع من فئات المجتمع الذي تخدمه ، ويعرف الإعلام المكتبي الرقمي على أنه " الإعلام الذي يعتمد على شبكة الإنترنت في التواصل والتفاعل مع المستفيدين ويستغل كل الإمكانيات والتقنيات والتطبيقات والتطورات الممكنة لنشر الرسالة 19 " الإعلامية وتوصيلها للمستفيدين وتلقي ردود الأفعال

وهناك عدة أشكال من وسائل الإعلام الرقمي الأي يمكن أن تستخدمه المكتبة الوطنية في التعريف بخدماتها من خلال موقعها الرسمي على شبكة الإنترنت من هذه الأثكال المستخدمة ما يلي : • • إستخدام المكتبة الوطنية الإعلام الرقمي للتعريف بخدماتها وإتاحتها رقمياً على موقع المكتبة على شبكة الإنترنت .

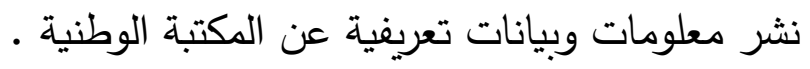
الإستفادة من كافة الإمكانات والتطبيقات والتقنيات لوسيلة الإعلان الرقمي للإعلان والتسويق عن خدمات المكتبة .

هبة محمد خليفة عبد العال ـ الإعلام المكتبي الرقمي في المكتبات الوطنية : دراسة مقارنة ـ إثر اف زين 19

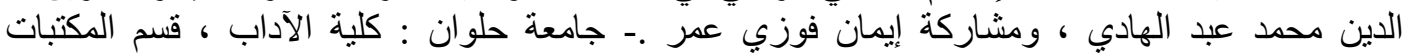

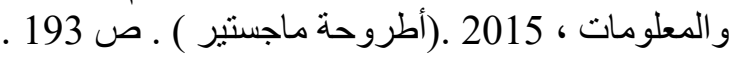


التحديث المستمر لبيانات المكتبة على وسائل الإعلام الرقمي .

\section{7 - خدمة محو الأمية المعلوماتية وتدربب المستفيدين :}

خدمة محو الأمية المعلوماتية هي إحدى الخدمات التي تعمل على توعية المستفيدين بالقدرات والمهارات التي تكفل لهم إدراك الحاجة إلى المعلومات ، وتحديد هذه المعلومات ، وتقييمها والإفادة منها بصورة فعالة ، حيث أن محو الأمية المعلوماتية متطلب رئيسي من متطلبات التعلم مدى الحياة Lifelong Learning الذي يضيف المهارات ذات Creation of وإعادة إنتاج المعلومات Interpretation الصلة بتفسير المعلومات 20 لصالح المنظمة التي يعمل بها المستفيد Information وتنقسم خدمة تدريب المستفيدين داخل المكتبة الوطنية إلى مستويين أساسيين : مستوى أساسي : وهو مخصص للمستفيدين الجدد من المكتبة الوطنية للتعريف بالمكتبة ومقتتياتها ، وسياسة الإعارة ، والفهرس الآلي ، والوصول إلى المصادر داخل المكتبة

مستوى متقدم : وهو مخصص للباحثين وطلاب الدراسات العليا للتعرف على قواعد المعلومات الإلكترونية التي توفرها المكتبة وطريقة إستخدامها . ويهدف التدريب والإرشاد إلى تعريف المستفيدين من المكتبة الوطنية بالآتي : 1- الإمكانيات المتاحة للحصول على المعلومات ، من خلال تعليم استخدام فهارس المكتبة ، واستخدام الكتب المرجعية وغير ذلك . 2- أفضل أساليب الحصول على المعلومات . 3- التعبير بدقة عن إستفسار المستفيد وتحديد مجال إهتمامه .

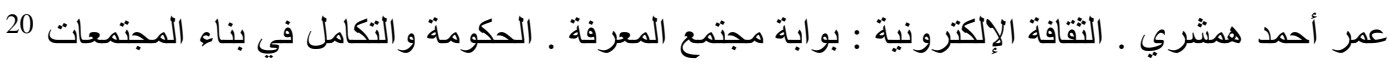

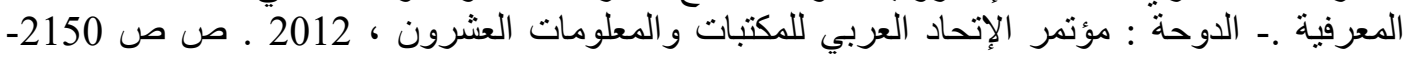
.2169 
4- إنجاز الأعمال والجهود العلمية بطريقة تكفل سهولة تجهيزها وتتظيمها من قبل

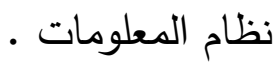

\section{8cientific Translation : خدمة الترجمة العلمية}

\section{: Service}

عرف حشمت قاسم الترجمة العلمية بأنها " ترجمة الوثائق أو النصوص الموضوعية أو التخصصية ، حيث تتم ترجمة الوثائق المتخصصة إستجابة لطلب المستفيدين بهدف تخطي أو إجتياز الحواجز اللغوية ، ويتركز الإهتمام في المقام الأول على المادة العلمية التي تثتمل عليها الوثائق المترجمة ، بينما يأتي الإهتمام بالثكل أو الأسلوب في المرتبة الثانية ، وذلك على عكس الترجمة الأدبية التي تهتم بصفة أساسية بجمال الأسلوب وروعة العبارة " 21 وقد إنتشرت الترجمة الإككترونية بدرجة كبيرة في ظل إستخدام شبكة الإنترنت ، وتوافرت العديد من المواقع التي تقدم ترجمة آلية مجانية عبر شبكة الإنترنت ، وتوفر بعض محركات البحث مثل Google خدمة الترجمة للصفحات التي تقدمها للمستقيدين ضمن نتائج البحث .

لذا كان لزاماً على المكتبة الوطنية أن تهتم بتقديم خدمة الترجمة العلمية الإلكترونية على موقعها الرسمي على شبكة الإنترنت لجمهور المستفيدين لمواكبة التطورات العلمية المتعلقة ببناء مجتمع المعرفة والإنفتاح على الثقافات والحضارات العلمية المختلفة بثتى اللغات من كل أنحاء العالم .

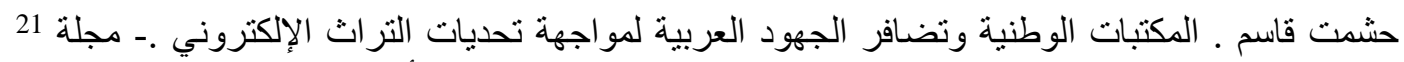

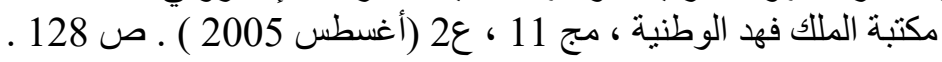




\section{9 - خدمة البحث والإسترجاع بقواعد البيانات :}

أصبح لزاماً على المكتبات الوطنية أن تقوم بالإشتراك أو بإنشاء قواعد البيانات

وإتاحتها للمستفيدين سواء عبر شبكة داخلية في المكتبة أو عبر موقعها على شبكة الإنترنت ، حيث أن قواعد البيانات تحتوي على مقالات الدوريات العلمية المُحكمة والرسائل الجامعية وبحوث المؤتمرات والكتب على المستوى العالمي وبصيغة إلكترونية ، وينبغي على المكتبة الوطنية أن تتعاقد مع ما هو مناسب لإحتياجات المستقيدين منها ومع ما يتتاسب مع ميزانية

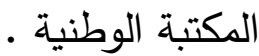

\section{0- خدمة النشر العلمي الإلكتروني على موقع المكتبة}

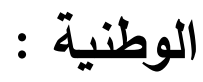

قدمت الويب إمكانات النشر العلمي الإكتروني للهيئات والأفراد على إختلاف مستويات وأوجه النشر الرقمي لمواد المعلومات العلمية أو الثخصية ذات الوسائط المتعددة وليست النصية فقط ، وقد عملت مواقع المكتبات الوطنية على إستخدام هذه الإمكانات في تقديم خدمات النشر العلمي الرقمي لمواد المعلومات المتخصصة والتراثية . وتُعرف خدمة النشر العلمي الإكتروني بأنها " أحد أشكال النشر الإكتروني لمصادر المعلومات ، غير أنه لا يهدف إلى الربح أو صناعة النشر التجاري ، وتقدم هذه الخدمة من خلال مواقع الهيئات العلمية أو مؤسسات المكتبات الوطنية على إعتبار أنها الهيئات المنوطة بإفادة الباحثين ونشر مصادر المعلومات العلمية دون هدف الربح أو التعامل التجاري مع مصادر المعلومات كسلع " .

سيد ربيع سيد إبراهيم .خدمات النشر العلمي على مواقع المكتبات الوطنية العربية : دراسة تطبيقية .ـ- 2012 . بحوث في علم المكتبات و المعلومات ، ع8 (مارس المات 2012) ـ ص 213 ـ . 
وقد حدد الباحث سيد ربيع أشكال المعلومات العلمية المنثورة على مواقع 23 (2) المكتبات الوطنية وهي كما يلي

* الفهارس : يعتمد هذا الثكل على قاعدة بيانات متاحة من خلال موقع الويب . " الكتب : يعتمد على ملفات Adobe Reader المقروءة بواسطة برنامج " الدوريات : من خلال موقع المكتبة وملفات PDF للمقالات داخل كل عدد. " البحوث : ملفات PDF تحوي المقالات والبحوث ذات القيمة المستديمة . . يختص أحدها بالدوريات والآخر بالناشريين PDF الأدلة : ملفات" * النشرات الإخبارية : مجموعات المعلومات الإعلامية التي تقدمها مواقع المكتبات

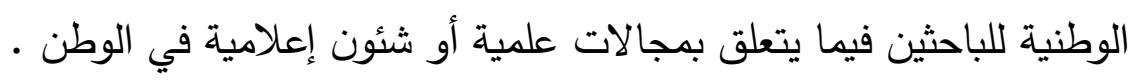
وترى الباحثة أن بيئة الويب عملت على تمكين مواقع المكتبات الوطنية الرقمية من

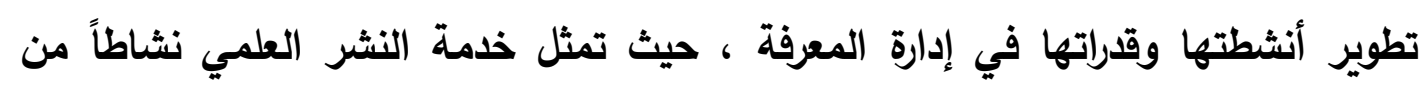
الأنثطة المستحثثة على مجال المكتبات وهو لعب دور الناشر العلمي للمواد المعلوماتية

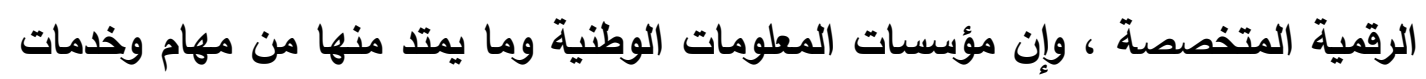

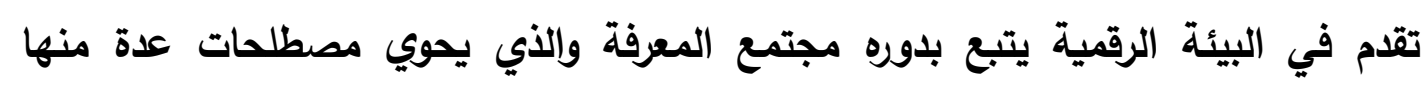
صناعة المعرفة في البيئة الرقمية من خلال مؤسسات معلوماتية وطنية قادرة على تفعيل العديد من الأنثطة والمهام المقدمة في شكل خدمات معرفية رقمية متطورة .

سيد ربيع سيد إبر اهيم ـ المرجع سابق . ص 229 .23 


\section{1- الخدمات المعرفية المستحدثة في ظل تطبيقات الويب 2}

: ( Web 2.0 )

في ظل تطبيقات الجيل الثاني للويب Web 2.0 أصبحت المكتبات أكثر فاعلية من خلال التحديث الدائم وتقديم الخدمات والتقنيات المساندة، والمستقيد هو المحور والأساس وبإمكانه الوصول إلى المعلومات في الزمان والمكان المناسبين له سواء أكان داخل المكتبة أو خارجها من خلال تطبيقات الجيل الثاني للويب Web 2.0 المختلفة كما أن أخصائي المعلومات ينبغي أن يكون ملماً بتقنيات الجيل الثاني للويب ومنفتح على أحدث تكنولوجيات

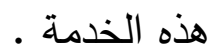

ومن أبرز تطبيقات Web 2.0 داخل المكتبات الوطنية والتي يمكن أن تُقدم كخدمة 24 معرفية متطورة ضمن خدمات المكتبة الوطنية ما يلي : (1) 1- المدونات الإكترونية Blogs : "وهي عبارة عن صفحات على الويب يمكن للجمهور استخدامها لإبداء تعليقاتهم ومشاركة أفكارهم وقد ينشئها فرد أو جماعة أو شركة تقدم من خلالها سجل نشاطاتها وأفكارها ، وتضيف المدونات تعليقات قصيرة وروابط خارجية على الإنترنت وتسمح للزوار بترك تعليقات على المحتوى للسماح بالتفاعل مع محرري هذه المدونات ، ويمكن للمكتبة الوطنية أن تستفيد من هذا التطبيق في مشاركة أحداث وفعاليات أنشطة المكتبة المختلفة مع جمهور المستثيدين منها ـ "

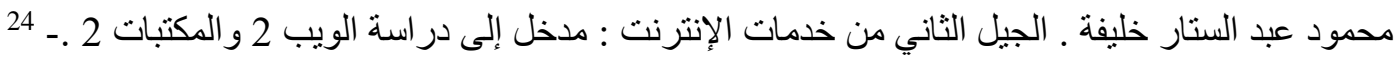

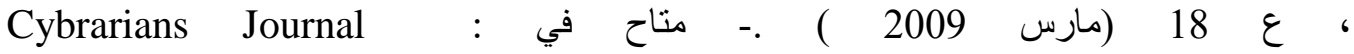
http://www.Journal.Cybrarians.org/index.php?option=com_content\&view cited in $2 / 12 / 2017$ 
2- الويكي Wiki : وهي عبارة عن مواقع تعاونية تمثل عمل جماعي لعدد من المؤلفين يسمح لهح بتحرير وتعديل المحتوى ، وتعد موسوعة Wikipedia من أشهر الموسوعات المستخدمة لهذا التطبيق ويمكن للمكتبة الوطنية أن تستفيد من تطبيق Wiki في إنثاء موسوعة وطنية أو دائرة معارف وطنية تؤرخ لتاريخ الدولة وثقافتها وحضارتها وتعمل بمثابة حافظة للتراث الوطني القومي للدولة .

3eally Simple Syndication (RSS) خدمة الملخص الوافي للمحتوى

وهي وسيلة لجمع موجز للأخبار والإعلانات والنشرات من مختلف المواقع الإلكترونية بما فيها مواقع المكتبات على شبكة الإنترنت ، ويمكن إستخدام هذه الخدمة داخل المكتبة الوطنية لبث المواد الجديدة التي تصل إلى المكتبة مثل الكتب والمواد السمعية والبصرية ،وأساليب إستخدام خدمة RSS إما بإستخدام قارئات الملخصات ، أو الإشتراك عن طريق التصفح مباشرة ومن أمثلة ذلك موقع Firefox و Safari Browser أو إستخدام • عSS

4- مواقع الشبكات الإجتماعية Social Networking Sites : وهي عبارة عن مواقع للتواصل الإجتماعي مثل (Facebook,Myspace) ، ويمكن للمكتبات الوطنية الإستفادة من هذه المواقع من خلال إنشاء حساب Account للمكتبة وإضافة الأصدقاء (المستفيدين) ، كما يمكن للمكتبات أن تقدم خدماتها المعرفية من خلال مواقع التواصل الإجتماعي مثل (خدمات الإحاطة الجارية ، الخدمات المرجعية ، إتاحة الأدلة وإرشاد وتوجيه المستفيدين حول كيفية إستخدام المكتبة ، أخبار المكتبة ، فعاليات المكتبة ، الأحداث القادمة وغير ها من الخدمات) 
5- خدمات الاردشة والمحادثة الفورية والمؤتمرات المرئية : وهي عبارة عن

خدمات وفرتها بيئة الجيل الثاني للويب يمكن من خلالها التواصل بين المستفيدين وأخصائيي المكتبات بشكل فوري عبر شبكة الإنترنت ، ويتم التواصل بالكتابة والصوت والصورة في وقت واحد ، كما يمكن من خلال هذه الخدمات تبادل الملفات الإككترونية ، ويتطلب إستخدام هذه الخدمات وجود أجهزة كومبيوتر أو هواتف ذكية وإتصال بشبكة الإنترنت ذات جودة عالية .

\section{2 - الخدمات المعرفية المقدمة من خلال تطبيقات الهواتف}

إقتحمت الهواتف الذكية Smart phones وتطبيقاتها مختلف مجالات الحياة بما فيها مجال المكتبات والمعلومات ، ومع ظهور الهواتف الذكية وتطبيقاتها برزت أمام المكتبات فرص حقيقية لتسهيل تقديم خدماتها المعرفية وإتاحة محتواها إلى المستفيدين منها ، Apple حيث أصبح بالإمكان الإستفادة من التطبيقات المتاحة على متجر كل من آي فون وأندرويد Google Play ، ففي العصر الذي نعيش فيه هنالك فئة عريضة من الجمهور تمتلك هذه الهواتف الذكية ومن الممكن إستهاف هذا الجمهور وتقديم الخدمات المعرفية لتعزيز تقديم المحتوى المعلوماتي ، وهناك عدد من تطبيقات الهواتف الذكية يمكن من خلالها تقديم الخدمات المعرفية لمجتمع المستقيدين من المكتبة الوطنية نذكر منها : 25 1- خدمة فهرس المكتبة المتكامل (الإعارة- الحجز ) : وهو عبارة عن تطبيق يسمح للمستخدمين الوصول إلى فهرس المكتبة من خلال هواتفهم الذكية ، وإستقبال الرسائل النصية SMS ، ووسائل الوسائط المتعددة MMS من المكتبة لإخطار المستفيدين بحالة المعلومات المطلوبة وإمكانية استعارتها أو حجزها .

مخلص محمد خير ـ دور المكتبات الجامعية في بناء مجتمع المعرفة ـ مرجع سابق . ص 156 ـ 25 
2- خدمة خريطة المكتبة التفاعلية Interactive Library Map : يساعد هذا

التطبيق المستقيدين على سرعة وكفاءة الوصول إلى موقع المصادر والوثائق داخل المكتبة ، ويقدم للمستفيدين الإتجاهات المحددة على الخريطة لترشدهم إلى مكان المصادر التي يحتاجونها ، وتتفاوت جودة هذا التطبيق حسب مزايا وقدرات الهواتف الذكية .

3- خدمة التوصية الآلية : Automated Recommendations : من خلال رسائل الوسائط والرسائل النصية التي ترسلها المكتبة للمستفيدين ، وذلك حسب إهتمامتهح الموضوعية وتخصصاتهم ، ويمكن للمكتبة معرفة هذه الإهتمامات من خلال مراجعة سجلات المستفيدين ومعرفة المصادر التي إستخدموها من قبل .

4- خدمة الحوسبة السحابية Cloud Computing : "هي تكنولوجيا تعتمد

على نقل المعالجة ومساحة التخزين الخاصة بالحاسب إلى ما يسمى بالسحابة ، وهي جهاز خادم يتم الوصول إليه عن طريق الإنترنت ، وبهذا تتحول برامج تكنولوجيا المعلومات من منتجات إلى خدمات ، وبذلك تساهم هذه التكنولوجيا في التخلص من مشاكل الصيانة ، وبالتالي يتركز مجهود الجهات المستفيدة على إستخدام هذه الخدمات فقط ، ويمكن للمكتبات الوطنية الإستفادة من هذا التطبيق من خلال النظم الآلية المتعلقة بالإعارة وإدارة المحتوى الرقمي والكتب الإكترونية .

من خلال ماسبق نجد أن محتمع المعرفة هو المجتمع الذي يتم فيه إنتاج

المعرفة بما يتناسب مع إحتياجات الإنسان ، وأن للمكتبات الوطنية دور هام حداً في بناء وتنمية محتمع المعرفة ، والتفكير المهني على مستوى المكتبات الوطنية وخدماتها ق تظور إلى درجة أصبح التغيير معها أمراً لالد منه ، وخاصة أنتا نعيش في عصر ثورة تكنولوحيا المعلومات والإتصالات ، كما أن الخدمات المعرفية أصبحت أكثر فعالية ومرونية Web 2.0 وسرعة من خلال تطبيقات مجتمع المعرفة ، مثل تطبقات الجيل الثاني للويب والكهواتف الأكية وغيرها. 


\section{سابعاً: الكوادر البشربة بالمكتبات الوطنية :}

إن توفير الكوادر البشرية المؤهلة والمدربة هي واحدة من المتطلبات الأساسية اللازمة لقيام مؤسسات المعلومات بدورها في مجتمع المعرفة بصفة عامة والمكتبات الوطنية بصفة خاصة ، بل هي أهم هذه المتطلبات ، فبدون العنصر البشري لن يكون هناك دور لمؤسسات المعلومات ، ولن تستطيع تحقيق أهدافها ، حتى ولو توافرت لها المتطلبات الأخرى من تكنولوجيا المعلومات والإتصالات، والتجهيزات المادية للمكتبة، والتمويل المالي الكافي . ولكي يؤدي أخصائي المعلومات المهام المّوكلة إليه بكفاءة وإقتدار ،فان ثمة كفاءات ومهارات وخبرات يجب أن يكتسبها ، ومن ذلك القدرة على التعامل مع متطلبات مجتمع المعرفة ، و تطبيق عمليات إدارة المعرفة - لاسيما - مع التطورات الحديثة لتكنولوجيا

المعلومات والإتصالات مثل تصميم وإنشاء قواعد البيانات ، وبناء المستودعات ، والبرمجة ،

وتتظيم المعلومات والبحث عنها وإسترجاعها في البيئة الرقمية ، وتحليل النظم وتطويرها ، وإدارة الثبكات ، وتطوير المواقع ، فضلاً عن معرفته بأساسيات التعامل مع شبكة الإنترنت والقدرة على تقديم تقنياتها ، والإحاطة بحقوق الملكية الفكرية ونظم أمن المعلومات وحمايتها . ويمكن القول إن منظومة الكوادر البشرية المؤهلة للقيام بدورها في بناء مجتمع المعرفة داخل المكتبة الوطنية يمكن تصنيفها إلى ثلاثة أقسام رئيسية وهي : 1 - مديرين المعرفة. 2- أخصائي المعرفة. 3- رأس المال المعرفي . 


\section{أولاً: مديرين المعرفة :}

يعد هذا المصطلح من أهم الصصطلحات البارزة في مجتمع المعرفة حيث يحفل Knowledge الإنتاج الفكري بالعديد من التعريفات لمصطلح مديرين المعرفة وصناعها 26 : ويككن أن نميز في هذا السياق ثلاثة اتجاهات رئيسية Workers ل الإتجاه الأول : يحصر عمال المعرفة في مستخدمي تكنولوجيا المعلومات

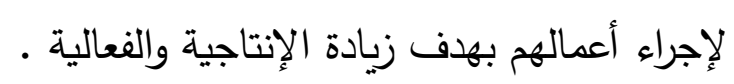
الإتجاه الثاني : يرى صناع المعرفة الأفراد الذين يضيفون القيمة للمنتجات والخدمات من خلال تطبيق المعرفة . الإتجاه الثالث : يرى الأفراد الذين يستخدمون عقولهم أكثر من أيديهج لإنتاج

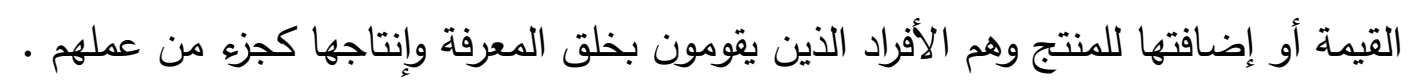

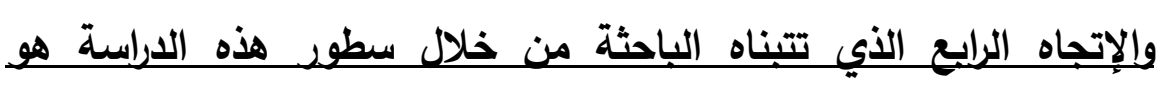

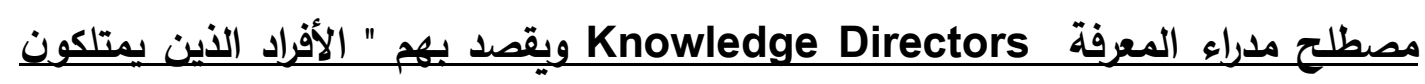
المعرفة ، ولديهيم القدرة على استخدامها وتطيقها ، ومعرفة متى يتم ذلك بهذف النجاح وزيادة الإنتاجية والنمو التنافسيـ"

وهناك مجموعة من المهارات الواجب امتلاكها من قبل مديرين المعرفة وهي تتمثل فيما يلي :

أ- - التفكير الإستراتيجي بما يضمن الرؤية الواضحة عن كيفية تحسين أداء المؤسسة

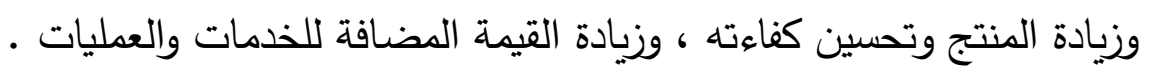

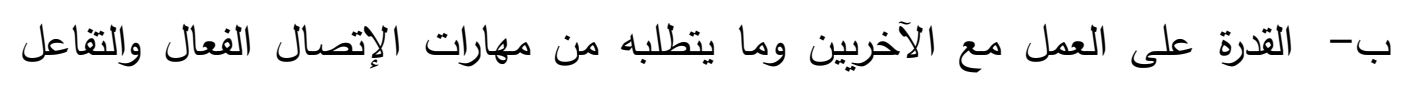
· الديناميكي معهم

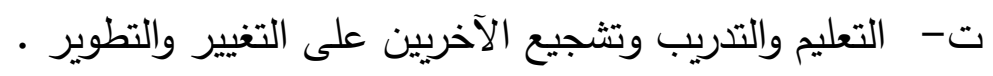

السيد السيد النشار ـ أساسيات إدارة المعرفة . مرجع سابق ـ ص26 .26 
ث- - الفهم العميق للأمور بما يتيح تشخيص المشكلات وتأثيراتها وكيفية حلها وإيجاد البدائل لها .

ج- المعرفة التامة بتكنولوجيا المعلومات وتطبيقاتها ونظمها .

ولذلك فإن مديرين المعرفة هم أساس نجاح أي مؤسسة لا سيما في البيئة الايناميكية المتغيرة مثل بيئة المكتبات ، والتي يحتاج العمل فيها بصفة مستمرة إلى لى الإبتكار والإبداع والأفكار الجديدة ، ومن ثم البحث والتنقيب المستمر في مستودعات المعرفة والإفادة منها ، وتوسيعها بالتعلم المستمر ، وليس مجرد تجميع مصادر المعلومات ،ومعالجتها ،وتقديم خدمات المعلومات التقليدية .

\section{ثانياً : أخصائي المعرفة داخل المكتبة الوطنية :}

أصبحت المعرفة أهم وسائل نهضة الأمم وتتميتها في جميع المجالات وذلك بعد أن

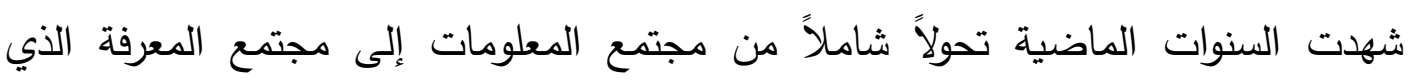
يتمحور حول بناء القدرات للبحث عن المعلومات وتنظيمها ومعالجتها وتحويلها واستخلاص المعرفة من أجل تطبيقها لأغراض التنمية الإنسانية ، وقد تطلب ذلك ضرورة أن تواكب

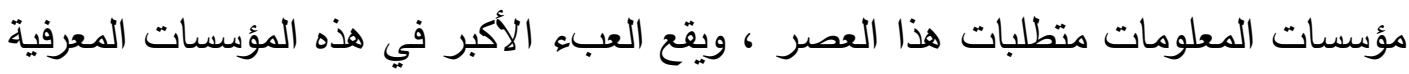

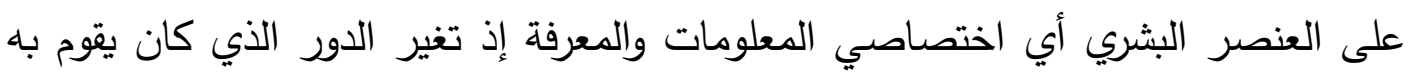

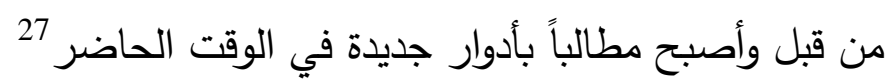

وهناك العديد من المسئوليات الملقاة على عاتق أخصائي المعرفة في عصر مجتمع

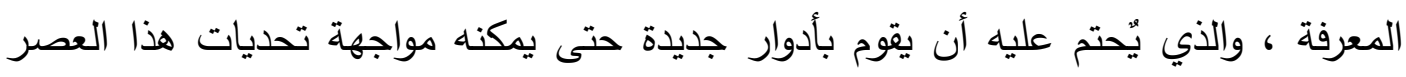

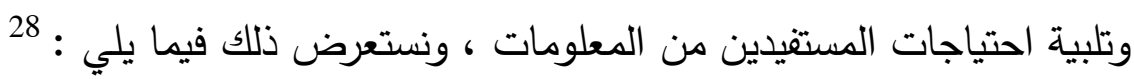

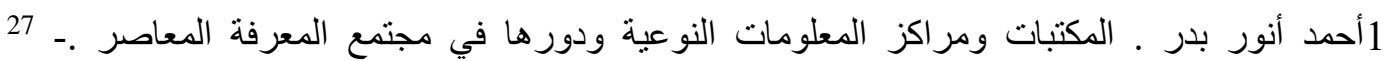

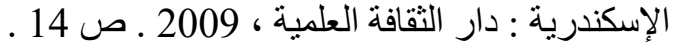

نبيل علي ـ إقامة مجتمع المعرفة كمور للنهضة .ـ المستقبل العربي ، ع 342 (أغسطس 2007 ) ـ ص ص ص 23 ـ 28

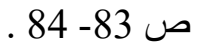


1- المساهمة بقوة في بناء العالم الرقمي : إن دور أخصائي المعرفة كمستلم لمصادر وأوعية المعلومات قد انتهى تماماً ، وهناك اتجاهاً متزايداً في عصر مجتمع المعرفة أن يساهم الأخصائي بدور فعال في إنتاج المعلومات والمحتوي الرقمي الخاص بالمؤسسة التي يتبعها.

ويمكن لأخصائي المعرفة داخل المكتبة الوطنية إنثاء المصادر الإكترونية للمعلومات ورقمنة محتويات المكتبة الوطنية المختلفة ، والأرشفة الرقمية لمصادر المعلومات التي تمتلكها المكتبة هذا بالإضافة إلى إنثاء وتصميم قواعد البيانات البيليوجرافية وقواعد بيانات النص الكامل وذلك بالتعاون والمشاركة مع أخصائيين تكنولوجيا المعلومات والحاسبات العاملين بالمكتبة مثل : مصمم الويب وقواعد البيانات وأخصائي الجرافيك واختصاصي المحتوى الرقمي وغيرهم . 2- المساهمة في ابتكار وتصميم النظم الآلية المتكاملة :

ليس من الملائم أن يُترك ابتكار النظم الآلية المتكاملة للمكتبات للمهندين وحدهم بل لابد أن يساهم اختصاصي المعرفة في بناء هذه النظم وليس تشغيلها او استخدامها فقط ، ومن المفيد جداً أن يسعى أخصائي المعرفة إلى تصميم موقع المكتبة على شبكة الإنترنت ، وإنشاء نظام آلي متكامل لإدارة عمليات المكتبة المختلفة - لا سيما أن تتظيم المعومات وبثها بشكل ملائم للمستفيدين هو من صميم اختصاصات أخصائي المعرفة في عصر مجتمع المعرفة المعة 
3- المساهمة في إدارة المشروعات الكبيرة للمكتبة الوطنية :

تتولي المكتبات الوطنية الآن العديد من المشروعات الجديدة المتعلقة باستخدام تكنولوجيا المعلومات في الأنشطة المختلفة ، وهذه المشروعات مثل : رقمنة الكتب التراثية ، إعداد قاعدة بيانات للخرائط ، رقمنة أوائل الدوبيات العربية ، إنشاء متحف للمواد النادرة بالمكتبة ، إعداد تسجيلات بيليوجرافية إلكترونية للرصيد الراجع بالمكتبة ، كل هذه المشروعات وغيرها تتكلف في الغالب مبالغ مالية كبيرة ، وقد يستعان في تغطيتها بمصادر خارجية ومن هنا يصبح من الضروري أن يتولى أخصائي المعرفة إدارة مثل هذه المشروعات بنجاح

\section{4- الإنخراط بنجاح في الأنشطة التعليمية والإجتماعية والثقافية للمكتبة :}

شهدت الفترة الحالية من عصر مجتمع المعرفة توسعاً وامتداداً واضحاً في نطاق أنشطة المكتبات ، فقد تحولت المكتبات العامة إلى مراكز مجتمعية وثقافية للمجتمعات التي توجد فيها ، وتحولت المكتبات المدرسية إلى مراكز مصادر التعلم ، كما انغست المكتبات الجامعية في مساعدة الجامعات في مبادرات التعليم الإلكتروني بأشكاله المختلفة ، وأصبحت المكتبات المتخصصة ومراكز المعلومات تؤدي دوراً فاعلاً في إدارة المعرفة في الثركات والمؤسسات التجارية وإلصناعية .

ويُمكن أن تساهم المكتبة الوطنية في إدارة مشروعات الدولة الرقمية لإتاحة المعرفة على المستوى الوطني بما تمتلكه من رصيد معرفي ضخم يمثل تراث الأمة الفكري وحضارتها ، ومثال على ذلك يُمكن أن تساهم دار الكتب القومية المصرية في مشروع بنك المعرفة المصري ذلك المشروع العملاق الذي يتيح مصادر معرفية عالمية للمستفيدين في جميع التخصصات والمجالات العلمية المختلفة ، ويتم ذلك من خلال رقمنة التراث الفكري الذي تمتلكه دار الكتب القومية المصرية عبر تاريخها الطويل من ( مخطوطات ، أوائل مطبوعات ، مصادر معلومات مختلفة في شتى فروع المعرفة البشرية ، وثائق ، ...الخ من 
مصادر المعلومات ) ، ويمكن أن يتم ذلك من خلال إنشاء بوابة إلكترونية باسم دار الكتب القومية وإتاحة تصفحها من خلال موقع بنك المعرفة المصري ، ذلك سوف يُسيهم بدوره في إعادة إحياء مكانة دار الكتب القومية بين جمهور المستفيدين منها .

ويوضح الثكل التالي المسميات الجديدة للمكتبات في عصر مجتمع المعرفة :

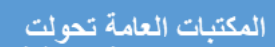 \\ لمر اكز مجتمعية وثقافية تمبرة}

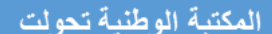

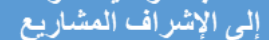

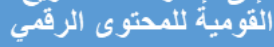

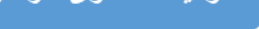

المكتبات الجامعية مركز

الثمبتم الإلكترونية مريز

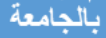

توالمكبات المدرسية

تحولت لمبر اكز لمصنادر

التطلم

\section{ثالثاً : رأس المال المعرفي بالمكتبة الوطنية :}

يشير هذا المصطلح إلى امتلاك المعرفة والخبرة التطبيقية والمهارات التخصصية

التي تزود بمجموعها المؤسسة بالحد التتافسي للسوق أي المعرفة التي تكون قيمة المؤسسة ، وتثتمل على ثلاثة مكونات رئيسية وهي : رأس المال البشري وهو المعرفة الموجودة في عقول العاملين بالمؤسسة ، والمهارات والخبرات التطبيقية التي يمتلكونها سواء أكانوا مبدعين أم أفراد عاديين إذ أن لكل منهم مساحته في المعرفة الضمنية تتناسب وإمكاناته ، والمكون الثاني أس المال الهيكلي وهو مجموعة الإستراتيجيات والهياكل والأبنية والنظم والبرمجيات والإجراءات والتجهيزات وغيرها من عناصر البنية التحتية ، وأخيراً أسس المال الزبائني ويقصد 
بها معرفة العملاء بالخدمات والأنشطة التي تتوفر المؤسسة عليها ومدى رضائهم عن مستوى

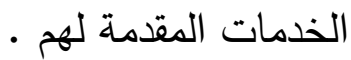

\section{وقد وردت في أدبيات الإنتاج الفكري تعريفات كثيرة ومتعددة لمصطلح رأس المال}

\section{المعرفي نذكر منها ما يلي : - المي}

O مدرة عقلية على توليد الأفكار المتعلقة بالتطوير الخلاق والإستراتيجي الذي

29.

O مجموعة الأصول المعرفية المعتمدة على العقول البشرية المبدعة م ومتطلبات ، ونظم العمل ، والعلاقة مع العملاء والتي تؤدي إلى الإنتاج المستمر للأفكار والأساليب الجديدة التي تحقق قيمة مضافة للمنظمة ، وتدعم قدراتها التنافسية ـ O مفوة العاملين الذين يمتلكون قدرات عقلية ومهارات بحيث يكونوا قادرين على إبداع وإنتاج أفكار جديدة قادرة على المحافظة على إبداع وإنتاج أفكار جديدة قادرة على الدحافظة على وضع الهيئة التنافسي ، وزيادة إنتاجيتها ، وتقليل التكلفة ، وتعظيم نقاط القوة داخل المؤسسة ، ولا يشترط توفر شهادة أكاديمية في رأس المال المعرفي، ولا تتحدد في مستوى إداري معين، ساعين من خلال ذلك لإقتناص الفرص، والمحافظة على المستفيدين. 31

خالد محمد طلال ـ تحليل معطيات العلاقة الإرتباطية بين نظام معلومات الموارد البشرية ورأس المال 29

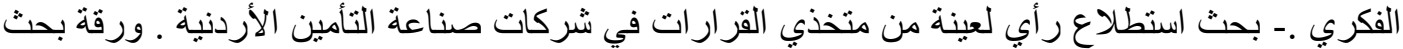

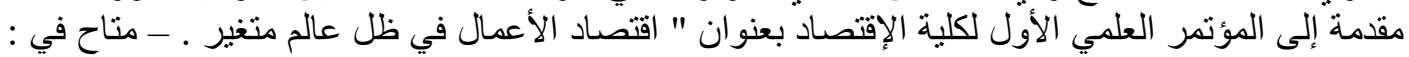
http://abufara.net/index.php/2012-10-21-10-21-10-57 cited in 25/4/2016

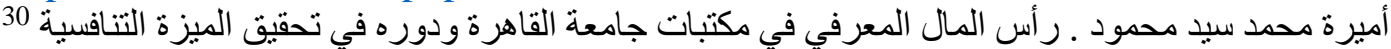

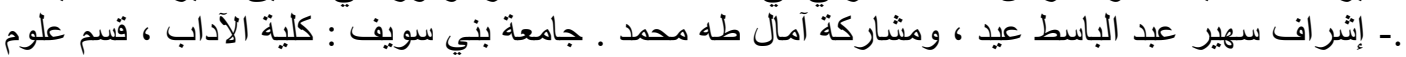

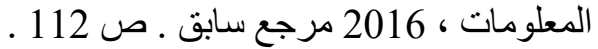

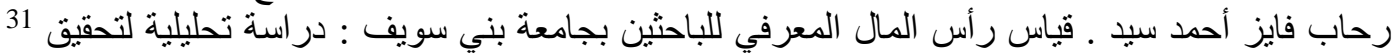

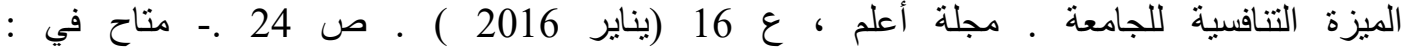
http://www.researchgate.net/profile/Dr rehab yousef/Publications cited in $\underline{25 / 2 / 2016}$ 
ومن التعريفات السابقة يمكن للباحثة أن تستخلص التعريف الإجرائى التالي لمصطلح رأس المال المعرفي : "عبارة عن امتلاك المنظمة نخبة متميزة من العاملين الأين يمتلكون مجموعة من القدرات المعرفية والتنظيمية دون غيرهم تمكنهم من إنتاج أفكار جديدة أو تطوير أفكار قديمة ، كما يمكن أيضا أن تسهم هذه القدرات في تحقيق الميزة التنافسية لمؤسساتهم ، ثم الإسهام في تطوير مجتمعاتهم " - أهمية تطبيق رأس المال المعرفي في المكتبة الوطنية : من الممكن توضيح أهمية تطبيق رأس المال المعرفي داخل المكتبة الوطنية من 32 خلال النقاط التالية

1. اتساع حجم المكتبات ومراكز المعلومات وزيادة الإنتاج الفكري فيها ، والذي يعتمد بشكل أساسي على قدرة المكتبات في إدارة رأس مالها البشري ، حيث أن المكتبات الناجحة هي التي تستطيع أن تجذب إليها الآخرين من خلال أفضل الخدمات التي تقدمها واستخدام ما تخزنه من معارف وقدرات .

2. زيادة القدرات الإبداعية للعاملين بالمكتبة وإبهار المستفيدين وجذبهم وتعزيز ولائهم • 3. إن المكتبات التي تتوي العمل في بيئة مجتمع المعرفة ينبغي أن تعتمد على استثمار إمكاناتها المعرفية لكي تنتقل من العمل التقليدي إلى العمل المعرفي الذي تكون فيه تكنولوجيا المعلومات أداة لتحقيق أهداف المكتبة وليس بديلا عنها . 4. إن رأس المال المعرفي يعد أهم مصادر الميزة التنافسية للمكتبات الحديثة إذ أن إستراتيجية التميز لا يمكن تحقيقها إلا من خلال النتاجات المعرفية المتمثلة في إبداع العاملين وتقديمهم لكل ما هو جديد في خدماتهم ووظائفهم •

زين الدين محمد عبد الهادي ـ رأس المال الفكري في المكتبات .- مجلة مكتبات نت ـ متاح في : 323 http://www.ipisegypt.com/articledetails.asp?MGID=8\&volID=43ARID=313 cited in $15 / 5 / 2017$ 


\section{ثامناً : المكتبات الوطنية الأجنبية والعربية ودورها في بناء}

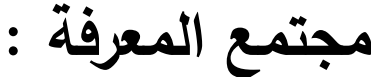

\section{أولاً: المكتبات الوطنية الأجنبية : مكتبة الكونجرس الأمريكي نموذجاً}

تعتبر مكتبة الكونجرس الأمريكية هي المكتبة الوطنية الرسمية للولايات المتحدة الأمريكية ، وهي تشغل مكانة فريدة في الحضارة الأمريكية ، وقد أُنشئت بموجب قانون الكونجرس الأمريكي في عام 1800 ، وقد تكلف إنشائها في ذلك الوقت 5000 دولار (وهو مبلغ هائل بالنسبة لتلك الحقبة الزمنية ) ، ولقد أصبحت مكتبة الكونجرس منذ منتصف التسعينات من القرن العشرين مصدراً دولياً غير محدود الأبعاد في تجميع مواد البحث العلمية في معظم اللغات والأشكال. 33

وتلك المكتبة العريقة الضخمة لا توازيها في نطاق خدماتها أي مكتبة وطنية أخرى حول العالم أجمع ، وقد أصبحت واحدة من المؤسسات الريادية في العالم للبحث والثقافة نظراً للإمكانيات المالية الضخمة المخصصة لتطوير تلك المكتبة ومواكبتها لعصر مجتمع المعرفة العالمي والمساهمة بقوة في إرساء أسس ودعائم ذلك المجتمع ، لذا كان لزاماً على الباحثة أن تتناول هذه المكتبة وتجربتها الفريدة في بناء مجتمع المعرفة الأمريكي والعالمي •

محمد فتحي عبد الهادي، نبيلة خليفة جمعة . المكتبات الوطنية ـ القاهرة : الدار المصرية اللبنانية ، 2009. 33

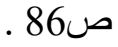




\section{- دور مكتبة الكونجرس في بناء مجتمع المعرفة :}

\section{أولأ: مشروع الذاكرة الأمريكية الوطنية : (المكتبة الوطنية الرقمية الأمريكية )}

يعد مشروع الذاكرة الأمريكية الوطنية الذي تبنته مكتبة الكونجرس الأمريكية في

American Digital عام1992 والذي أخذ مسمى المكتبة الوطنية الرقمية الأمريكية National Library وذلك لما به من مصادر معلومات مفيدة ومجموعة عالمية تتميز بأنها تجمع المعرفة على مستوى العالم وتقدمها للشعب الأمريكي بوجه خاص والعالم بوجه عام 34

\section{وهناك مجموعة من الأهداف التي سعى المشروع الأمريكي الوطني إلى تحقيقها}

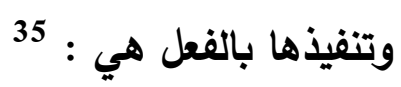

• عفظ وتخزين الوثائق التاريخية الأمريكية المميزة وجعلها متاحة على شبكة الإنترنت لكل المستخدمين في كافة أنحاء العالم .

• عرض أكثر من 7 ملايين مادة رقمية في أكثر من مائة مجموعة تاريخية على موقع المكتبة الرسمي على شبكة الإنترنت . • يقدم موقع المكتبة مجموعة من الخدمات البحثية للمستقيدين في تخصصات مختلفة مثل الزراعة واللغات والأدب والفن • تقدم المكتبة الرقمية الوطنية الأمريكية إمكانية استخدام الخدمة المرجعية الرقمية والتفاعل مع أخصائي المكتبة .

${ }^{34}$ 33Marchionini,G and Catharine Plaisant. Interfaces and Tools for the Library of Congress National Digital Library Program .- Elsevier Science, Information Processing and Management, vol 34 (no 5) (1998). Pp 535-555

3534 Rajkumar, J . ETANA-CMV: A Coordinated Multiple view visual browsing Interface for ETANA- DL .Thesis (Msc).- Virginia Polytechnic Institute and State University . p 230 . 


\section{ثانياً: برنامج الولايات المتحدة الوطني للبنية التحتية الرقمية

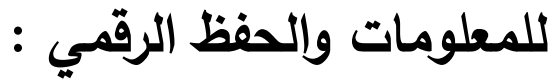

استكمالًا لجهود مشروع المكتبة الوطنية الرقمية الأمريكية السابق ونجاح هذا

المشروع، قامت الولايات المتحدة الأمريكية بوضع برنامج وطني للبنية التحتية الرقمية للمعلومات والحفظ الرقمي وقد وقع إختيار الحكومة الأمريكية على مكتبة الكونجرس الأمريكية ومجلس المكتبات ومصادر المعلومات الأمريكي وأيضاً إختيار أربعة دول أجنبية خارج نطاق أمريكا الثمالية تلك الدول هي (أستراليا - المملكة المتحدة - فرنسا - هولندا) وذلك في عام 2003 من أجل التعرف على المبادرات الوطنية للحفظ الرقمي لهذه الدول وكان من نتائج هذا البرنامج ما يلي 36

وجود اختلاف كبير بين مبادرات الحفظ الرقمي من حيث كم ونوع مصادر المعلومات الرقمية وضعف المخصصات المالية اللازمة لتمويل مشروعات الحفظ الرقمي على المدى الزمني الطويل في المكتبات الوطنية الأجنبية سالفة الذكر ، وتأكيد هذه المبادرات على أهمية المشاركة في المصادر الرقمية عن طريق المشابكة الإكترونية وإتجاه هذه المبادرات إلى تيسير الوصول للمعلومات الرقمية بدلاً من إقتناء المصادر الرقمية عن طريق الحصول على تراخيص لإستخدام هذه المصادر عن بعد.

35 Neil Beagri. National Digital preservation Initiatives : An Overview of Developments in Australia ,France,the United Kingdom and Netherlands.Washington: The Council on Library and information Resources and the Library of congress, 2003 Available at http://www.clir.org/pups/reports/pup116/contents.html cited in 2/8/2016 
ولاحظت الباحثة أن المشروع السابق ما هو إلا برنامج تعاوني من قبل مكتبة الكونجرس الأمربكية مع غيرها من المكتبات الوطنية الأخرى في أربعة دول أوروبية مختلفة مما يكثف عن دور المكتبة الوطنية الأمريكية الريادي في تبادل الخبرات وتقديم الدعم المالي والتعاون مع مثيلاتها من المكتبات الوطنية الأجنبية وهي بذلك تحقق إحدى إستراتيجيات المكتبة الوطنية تجاه بناء مجتمع المعرفة من خلال التعاون وتبادل المعارف والخبرات بين المكتبات وبعضها البعض •

\section{World Digital الثاً: مشروع المكتبة الرقمية العالمية}

\section{(www.wdl.org) :Library}

إن مشروع المكتبة الرقمية العالمية هو أحد المشروعات الرائدة والهامة التي تبنتها ، مكتبة الكونجرس الأمريكية وسعت من خلالها إلى تحقيق مجتمع المعرفة الرقمي العالمي وترجع نشأة المكتبة الرقمية العالمية إلى إقتراح جيمس بيلنجتون أمين مكتبة الكونجرس الأمريكية الذي تقدم به في خطاب ألقاه أمام لجنة الولايات المتحدة الوطنية لليونسكو في يونيو عام 2005 ، وقد كانت الفكرة الأساسية هي وضع مجموعة من ثروات العالم الثقافية على شبكة الإنترنت بحيث يسهل الوصول إليها حتى تعكس إنجازات كل الدول والثقافات وتبرزها ، وتعزز بذلك الوعي والتقاهم بين الثقافات ، وقد رحبت منظمة اليونسكو بالفكرة كإسهام نحوتحقيق أهداف اليونسكو الإستراتيجية والتي تتضمن تعزيز مجتمعات المعرفة وبناء القدرات في الدول النامية ، وتعزيز التنوع الثقافي على الثبكة العنكبوتية ، وقد هدف هذا المشروع 37 إلى ما يلي

تعزيز التفاهم بين الدول والثقافات . توسيع حجم المحتوى الثقافي على الإنترنت كماً ونوعاً.

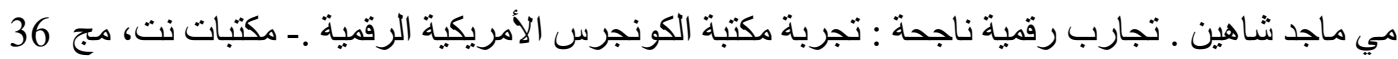

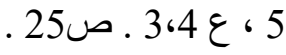


توفير مصادر معلومات للتربويين والباحثين والجمهور العام • بناء القدرات للمؤسسات الثربكة لتضييق الفجوة الرقمية بين الدول .

WDL وقد أتاح هذا المشروع المعرفي الرقمي المتمثل في المكتبة الرقمية العالمية مجموعة من المزايا لجمهور المستفيدين عبر العالم تمثلت فيما يلي : (1) 38 1. بيانات تعريف موحدة : كل مادة موصوفة بمجموعة محددة من البيانات البيليوجرافية (أو بيانات التعريف ) تتعلق بمكان وزمان وموضوع تلك المادة ، وتوفر بيانات التعريف المتسقة الأساس لموقع إلكتروني جذاب وسهل التصفح ، وتساعد على كثف أي صلات بين المواد المختلفة ، كما تُحسن بيانات التعريف المتسقة قدرة محركات البحث الخارجية على العثور على المادة موضوع البحث .

2. الوصف : من أكثر الميزات المثيرة للإعجاب في المكتبة الرقمية العالمية الأوصاف المقترنة بكل مادة ، التي كُتبت لتجيب على السؤاليين الآتيين : ما هي هذه المادة ؟ وما هي سبب أهميتها ؟ ، وهي مصممة لإثارة حب الإستطلاع لدى الطلاب وعموم القراء لمعرفة المزيد عن التراث الثقافي لكل الدول • 3. تعددية اللغات : بيانات التعريف وتعليمات التصفح والمحتوى الرقمي على الموقع مُترجم إلى سبع لغات : العربية ، الصينية ، الإنجليزية ، الفرنسية ، البرتغالية ، الروسية ،

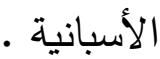

4. التطوير الفني للمكتبات الرقمية : لقد قدم فريق عمل المكتبة الرقمية العالمية أحدث التقنيات والتطورات في مجال الفهرسة وتطوير مواقع الإنترنت متعددة اللغات.

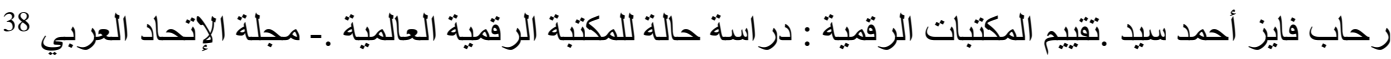
للمكتبات و المعلومات ، ع 17 ـ (يونيو 2016 ) ـ ص لص لر 163 


\section{ثانياً : المكتبات الوطنية العربية : مكتبة الملك فهر الوطنية}

\section{(السعودية) نموذجاً}

يرجع تاريخ المكتبة القومية السعودية إلى إنثاء مكتبة من جانب المواطنين في مدينة

الرياض بمناسبة تولي الملك فهد الحكم في البلاد ، وقد أطلق على المكتبة اسم (مكتبة الملك فهد ) ، وبدأ بناء المكتبة عام 1986 م (1406 هجرية ) تحت إشراف أمانة مدينة الرياض ، ثم طُرح إقتراح في عام 1988 م (1408 هجرية) يتضمن الإستفادة من مشروع مكتبة الملك فهد العامة وتحويلها إلى مكتبة قومية وتخصيص ميزانية مستقلة لها ، وقد تضمن الإقتراح 39 تغيير اسم المكتبة إلى ( مكتبة الملك فهر الوطنية ) .

- المهام والوظائف الرئيسية لمكتبة الملك فهر الوطنية :

قامت مكتبة الملك فهد الوطنية منذ الأيام الأولى بوضع إستراتيجية واضحة ومحددة ساعدتها في تحقيق مجتمع المعلومات داخل المملكة العربية السعودية ومن ثم التقدم بخطى ثابتة نحو التحول إلى مجتمع المعرفة وبناءه داخل المملكة على أسس قوية وسليمة هذه المهام والوظائف الرئيسية تمثلت فيما يلي : 40 جمع كل ما ينشر داخل المملكة العربية السعودية . جمع كل ما ينشره السعوديون خارج المملكة . جمع المواد العلمية من خارج البلاد المتعلقة باهتمامات المملكة ومواطنيها . جمع مصادر المعلومات التي سوف تساعد البحث العلمي ودراسة وفهم الحضارة البشرية في كل الإتجاهات .

جوز اء بنت محمد القحطاني ـ دور مكتبة الملك فهد الوطنية في بناء مجتمع المعرفة .- مجلة مكتبة الملك 39

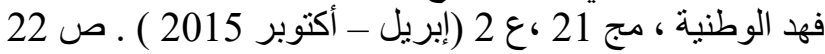

محمد فتحي عبد الهادي ، نبيلة خليفة جمعة . المكتبات الوطنية . مرجع سابق . ص ص ص 158 -159 ـ4 
جمع الكتب القديمة والنادرة وأوائل المطبوعات ، والمخطوطات والوثائق والرسوم والخرائط واللوحات المتعلقة بالحضارة الإسلامية والعربية .

إعداد ونشر البيليوجرافية الوطنية ، والفهرس الوطني الموحد ، والأدوات البيليوجرافية

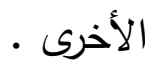

$$
\text { • إنشاء قواعد وشبكات معلومات . }
$$

تقديم خدمات مرجعية للوزارات والمؤسسات الحكومية ودعم الدراسات البحثية على نفقتها .

تتظيم وإستضافة مؤتمرات المكتبات والندوات ومعارض وأسواق الكتب . تمثيل المملكة في التجمعات الدولية المرتبطة بأهدافها ووظائنها . تبادل المطبوعات والمعلومات مع المكتبات الأخرى . التعاون مع الجمعيات والمنظمات الدولية في تخصص المكتبات والمعلومات . المشاركة الفعالة في تنمية خدمات المكتبات والمعلومات في المملكة العربية السعودية عن طريق : (1) أ- إعداد خطة وطنية لتنمية خدمات المكتبات والمعلومات بالتعاون مع المكتبات

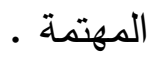
ب-تطوير المعايير البيليوجرافية الوطنية بالتعاون مع الكيانات المرتبطة ، وتقديم الدعم لتبني هذه المعايير ومتابعة تطبيقها ـ ت-تطوير البرامج لإستثمار المصادر المعلوماتية والمعرفية بما فيها إنثاء شبكة معلومات وطنية بالتعاون مع المكتبات ومراكز المعلومات الأخرى في المملكة نشر الإنتاج الفكري في علم المكتبات والمعلومات والموضوعات المرتبطة به . 


\section{الخدمات التي تقدمها مكتبة الملك فها الوطنية من أجل بناء مجتمع المعرفة : 41}

أولاً : الفهرس الإكتروني العام المتاح على موقع المكتبة على شبكة الإنترنت:

هي خدمة تقدمها المكتبة لجمهور المستفيدين منها من خلال الموقع الرسمي

للمكتبة حيث يتيح هذا الفهرس الإكتروني البحث وفقاً للمداخل التالية :

1- الكثاف العام لمصادر المعلومات الموجودة بالمكتبة .

2- 2 الكلمات المفتاحية للعنوان - 2

3- الكلمات المفتاحية للمؤلف .

4- الكلمات المفتاحية للموضوع •

5- البحث المتقدم Advanced Search

ثانياً: الخدمات الإكترونية المقدمة لجمهور المستفينين والباحثين :

* إمكانية طلب تسجيل مادة مطبوعة (للأفراد - دور النشر ) * طلب تسجيل مادة مطبوعة ( الجهات الحكومية ) * طلب تسجيل مادة غير مطبوعة . * طلب إفادة عن موضوع بحث ( ماجستير - دكتوراه )

* طلب قائمة مصادر حول موضوع ما باللغة العربية أو اللغة الأجنبية . * طلب مخطوطة أو وثيقة تاريخية . * طلب صور تاريخية متاحة بالمكتبة . * طلب اقتراح شراء كتاب أو موضوع محدد .

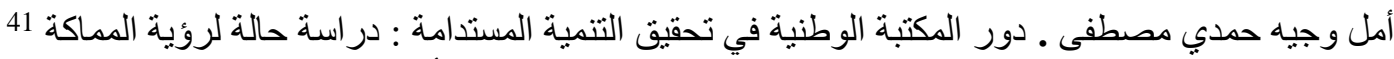

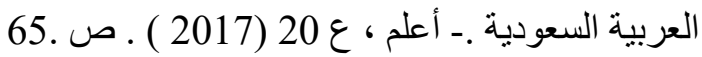




\section{* خدمة إعداد الملخصات للأبحاث والمصادر العلمية :}

وهي خدمة هامة جدا تقدمها المكتبة لجمهور المستفيدين منها عن طريق تصميم الملخصات العلمية للأعمال الفكرية التي يحثون عنها مع إتاحة الفرصة للمستفيدين للقيام بتقييم هذه الملخصات العلمية وتصويت الرأي حول نظام هذا التلخيص ومدى جودته ودقته * الخدمة المرحعية الرقمية من خلال موقع المكتبة على شبكة الإنترنت :

و إمكانية التواصل مع أخصائي المكتبة على مدار اليوم وتوجيه أي إستفسار إليه من خلال خدمة الرد على الإستفسارات وتلقي تساؤلات المستفيدين عبر موقع المكتبة الإلكتروني، والرد إلكترونياً على تلك الإستفسارات وتلقي المستفيد الأجوبة التي يبحث عنها داخل المكتبة.

\section{* إتاحة قواعد البيانات والمعلومات العالمية في جميع مجالات المعرفة البثرية :}

في إطار حرص مكتبة الملك فهد الوطنية على توفير أحدث مصادر المعلومات وتتظيمها وإتاحتها للباحثين، وكأحد أهدافها الرئيسة، فإن المكتبة وخلال عام 2015م أنهت الاشتراك في نحو 100 قاعدة بيانات الكترونية عربية وأجنبية للدوريات والكتب والرسائل العلمية، حيث سعت المكتبة إلى توفير مجموعة من قواعد لأهم ناشري ومزودي خدمات قواعد المعلومات مثل مجموعة قواعد Emelard ، و Ebsco، : Proquest المنظومة وغيرها من القواعد. وتغطي تلك القواعد مختلف مجالات المعرفة، وهي تشمل في تغطيتها كل الاختصاصات العلمية الرئيسية والفرعية، كالطب والقانون والهندسة والحاسب والعلوم الإنسانية والاجتماعية والتربوية إضافة إلى مجال الأعمال والاقتصاد وذلك دعماً لعملية البحث العلمي في المملكة العربية السعودية. ويمكن للباحثين الاستفادة من محتويات هذه القواعد من خلال زيارة المكتبة أو الاستفادة من خدمة) طلب قائمة مصادر (التي تتيحها المكتبة للباحثين دون الحاجة لزيارة المكتبة. 
* خدمة توثيق الإنتاج الفكري السعودي رقمياً( مشروع المكتبة الوطنية الرقمية

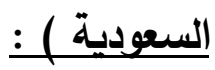

ينصب هذا المشروع على حصر مصادر المعلومات الوطنية المتخصصة في مجالات العلوم والتقنية، وتحديد حقوق الملكية الفكرية لتلك المصادر، وحفظها، وتنظيمها، وإتاحتها بنصوصها الكاملة عبر مكتبة رقمية على الإنترنت لعموم الباحثين والمستفيدين ودون

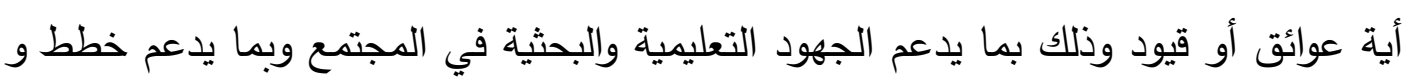

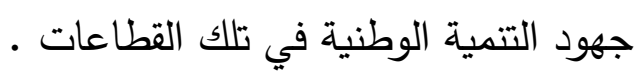
* خدمة الإعلام المكتبي الرققي

وهي خدمة تهتم بعرض أخبار وأنثطة وفعاليات المكتبة إلكترونيا للمستفيدين عبر موقع المكتبة على شبكة الإنترنت مثل ( إقامة المعارض والندوات العلمية والثقافية والمهرجانات والمؤترات العلمية الدولية ) وعرض تقارير موجزة عن هذه الأنشطة والفعاليات والتعريف بها .

\section{* خمة نشرة أخبار المكتبة :}

هي خدمة تقدم بشكل منتظم مرة واحدة شهرياً لجمهور المستقيدين من خدمات

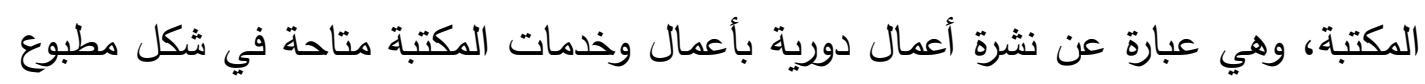
وأيضاً شكل إلكتروني يوجد بها معلومات عن ( أحدث الإصدارات التي تم إيداعها بالمملكة ،

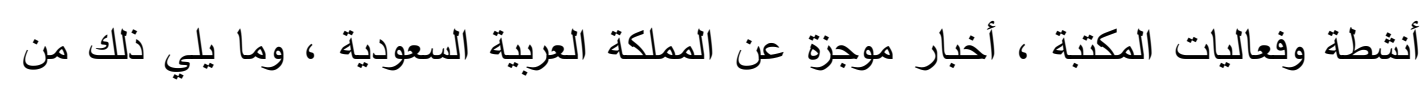
أخبار عامة ...... 


\section{*ذمة إصدار مجلة مكتبة الملك فهز الوطنية :}

وهي دورية علمية محكمة تهتم بنشر قضايا المكتبات والمعلومات في المملكة العربية السعودية والوطن العربي بأكمله وعرض الإتجاهات الحديثة في مجال المكتبات والمعلومات وإتاحة هذه الدورية إلكترونياً بالمجان لجميع المتخصصين في مجال المكتبات والمعلومات ، وهي بذلك تقوم بدور الناشر العلمي المتخصص في مجال المكتبات وتكنولوجيا المعلومات دون البحث عن أي ربح مالي ·

\section{* خمة طلب تدريب تعاوني}

تتيح هذه الخدمة للمستفيد تقديم طلب للتدريب التعاوني في المكتبة الوطنية ، وذلك سعياً منها لتحقيق رسالتها وأهدافها في المساهمة بالخدمة الاجتماعية بالشراكة مع الجامعات المحلية من خلال إعداد كوادر متميزة وذلك بإعطاء الطلبة والطالبات فرصة التدريب التعاوني في مختلف الإدارات في المكتبة حسب التخصصات المطلوبة . 


\section{تاسعاً : نتائج وتوصيات الاراسة :}

ــافت هذه الدراسة إلى التعرف على الخدمات المعرفية المتطورة التي ينبغي للمكتبات تقديمها في عصر مجتمع المعرفة بصفة عامة مع التركيز على دور المكتبات الوطنية في تقديم تلك الخدمات المعرفية بصفة خاصة ، وفي هذا السياق توصلت الدراسة الحالية إلى مجموعة من النتائج وهي :

1. ظهور مصطلح مجتمع المعرفة لأول مرة في ديسمبر 2003 م عقب القمة العالمية للمعلومات التي انعقدت بجينيف في هذا التاريخ ، والذي أكدت القعة أن المرحلة التي معيد

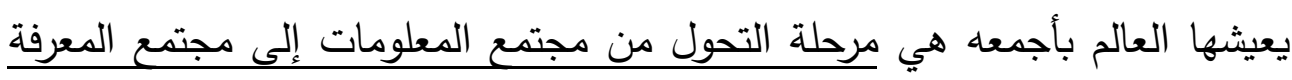
وعرفت القمة مصطلح مجتمع المعرفة بأنه " جميع الأنثطة والممارسات المرتبطة هينة

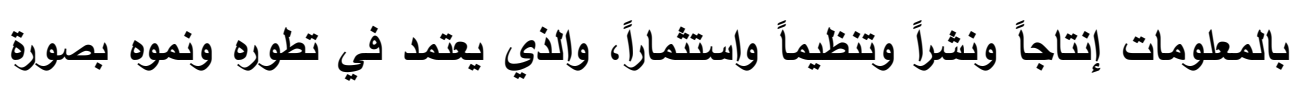

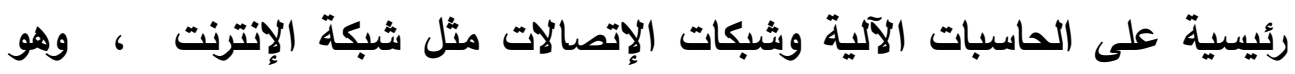

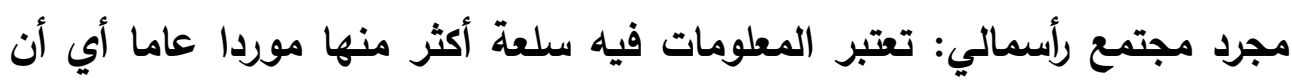

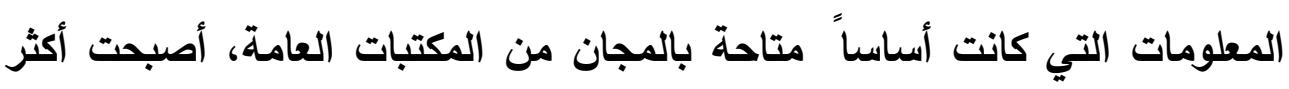
تكلفة عند الحصول عليها خصوصا بعد القيام بتخزينها في النظم المعتمدة على بلى بلى

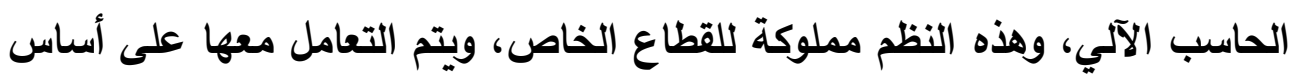

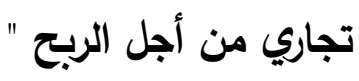
2. يُشكل العنصر البشري قيمة مميزة لاى مجتمع المعرفة إذ يمثل عاملي الإبداع والإبتكار ، ويتصف مجتمع المعرفة بتوفير مستوى عالي من التعليم والبحث العلمي لضمان خلق قوي بشرية مدربة ومؤهلة على أساسيات التحول لمجتمع المعرفة . 3. مجتمع المعرفة هو مجتمع الذين يعرفون ؛ والذي يعرف هو الثخص الذي يعرف ماذا يريد ؟ وكيف يصل إلى ما يريد؟ وكيف يوظف المهارات التي يمتلكها في تحقيق ما يريد ؟ 
4. المقومات الرئيسية الخمسة التي يستند إليها مجتمع المعرفة هي (الوعي المعرفي الكامل لدى أفراد المجتمع ، القوانين والتشربعات واللوائح المنظمة للعمل داخل بيئة مجتمع المعرفة ، وتوافر البنية التحتية لنظم الإتصالات وتكنولوجيا المعلومات بالدولة مروراً بالقيادة الإدارية الناجحة التي تتولى وضع الأسس وتوفير مقومات التنفيذ للخطط والبرامج المعرفية )

5. وضعت الباحثة تعريفاً إجرائياً للمكتبة الوطنية في عصر مجتمع المعرفة وهو " المكتبة التي تقتني كافة مصادر المعلومات التي تصدر داخل الدولة سواء المطبوعة أو الرقمية ، وتقوم برقمنة جمبع مصادر المعلومات المطبوعة إلى الثكل الرقمي سعياً منها إلى أن تصبح مكتبة وطنية رقمية بالارحة الأولي ، ثم تطق إدارة المعرفة على جميع العمليات الفنية ، كما أنها تقوم بتقديم مجموعة من الخدمات المعرفية المتطورة لجمهور المستفيدين منها ، وتسعى إلى مشاركة مصادرها المعرفية على المستوى المحلي والمستوى الدولي أيضاً تحقيقاً لمبدأ مشاركة المعرفة " 6. الخطوات التي ينبغي أن تتبعها المكتبات الوطنية لبناء مجتمع المعرفة هي : تجميع مصادر المعرفة البشربة ، إدارة المعرفة بالمكتبة الوطنية ،التحول للمكتبة الوطنية الرقمية ، تقديم مجموعة من الخدمات المعرفية المتطورة التي إرتبط ظهورها بالطفرة الهائلة في تكنولوجيا المعلومات والإتصالات وظهور الجيل الثاني من تطبيقات الويب وتطبيقات الهواتف المحمولة الذكية وغيرها مثل ( الموقع الرسمي للمكتبة الوطنية على شبكة الإنترنت ، الفهرس الإكتروني المتاح على الخط المباشر OPAC ، الخدمة المرجعية الرقمية ، خدمة الإمداد بالوثائق ، خدمة الإعلام المكتبي الرقمي ، خدة البحث والإسترجاع بقواعد البيانات العالمية ، خدمة النشر العلمي الإلكتروني على الموقع الرسمي للمكتبة الوطنية) . 
7. في عصر مجتمع المعرفة تحولت المكتبات بمختلف انواعها لتواكب هذا

العنصر كما يلي : (المي

أ- المكتبات العامة تحولت إلى مراكز مجتمعية وثقافية للمجتمعات التي توجد فيها

ب- المكتبات المدرسية تحولت إلى مراكز لمصادر التعلم •

ج- المكتبات الجامعية تُساعد الجامعات في مبادرات التعليم الإلكتروني بأشكاله

المختلفة .

د- المكتبات المتخصصة تؤدي دوراً فاعلاً في إدارة المعرفة في الشركات

والمؤسسات التجارية والصناعية .

هـ - المكتبة الوطنية في عصر مجتمع المعرفة يُمكن أن تساهم في مشروعات

الدولة الرقمية لإتاحة المعرفة على المستوى الوطني ، مثل مشروع بنك المعرفة المصري ،

والتي ينبفي أن تُشرف عليه دار الكتب القومية المصرية .

المكتبات العامة تحولتِ

لمر اكزب مجتمعية وثمقافية
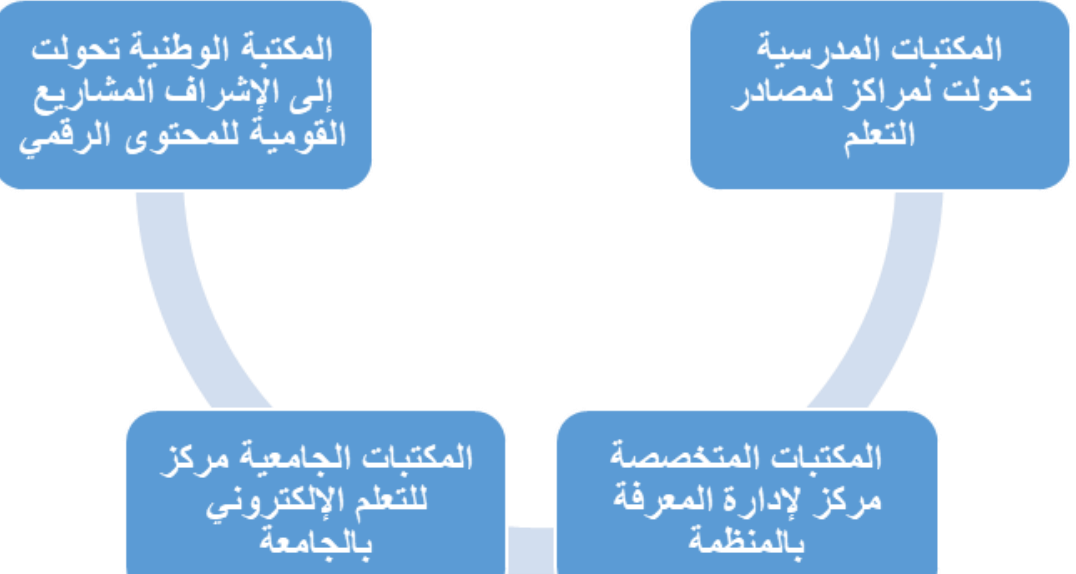

المكتبات المتخصصة المعرفة المبرة

مركز لإدارة المعرفة المبنة

بالامنظمة 
الموارد المعرفية للمكتبة الوطنية أربعة أنواع تتمثل فيما يلي :

1- معرفة العاملين : المعرفة الموجودة في عقول العاملين في المكتبة ، والمهارات

والخبرات التي يمتلكونها سواء أكانوا مبدعين أم أفراد عاديين ، إذ أن لكل منهم مساحة في المعرفة الضمنية تتناسب وإمكاناته.

2- المعرفة الهيكلية : وهي الأبنية ،والنظم ، البرمجيات، الإجراءات، التجهيزات ، وغيرها من عناصر البنية التحتية الداعمة للعاملين على القيام بمهامهم وتحقيق أهداف المكتبة

3- معرفة المستفيدين : وهي المعرفة عن المستفيدين الحاليين والمتوقعين

واحتياجاتهم ، ومدى رضاءهم عن الخدمات التي تتوفر المكتبة على تقديمها .

4- معرفة المؤسسات المنافسة : وهي المعرفة عن الخدمات والأنشطة والتسهيلات

التي توفرها المكتبات الأخرى وموردي المعلومات .

أما عن توصيات الدراسة الحالية فيمكن لنا إيحازها فيما يليـ

توصي الباحثة بعدد من المحاور التي يمكن تتاولها في دراسات مستقبلية مثل :

دور المكتبات الجامعية المصرية في بناء مجتمع المعرفة .

دور المكتبات العامة في بناء مجتمع المعرفة .

الخدمات المعرفية المتطورة المقدمة في المكتبات ومرافق المعلومات في

عصر مجتمع المعرفة .

مراجعة علمية للإنتاج الفكري حول إثكاليات وقضايا مجتمع المعرفة . مجتمع المعرفة كمقرر دراسي بأقسام المكتبات والمعلومات المصرية 


\section{مراجع ومصادر الدراسة \\ أولاً : المراجع العربية :}

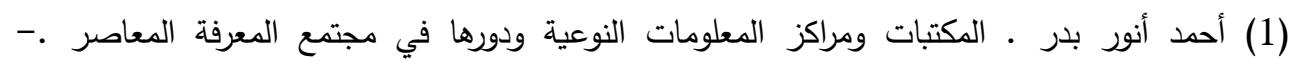
الإسكندرية : دار الثقافة العلمية ، 2009 ـ ص 14 .

(2) أحمد حسين بكر المصري ـ الفهارس الإجتماعية المتاحة على الخط المباشر :دراسة تحليلية للمتطلبات

الفنية والوظيفية لتصميم نموذج عربي .- جامعة حلوان : كلية الآداب ، قسم المكتبات والمعلومات ، 2013 .

$$
\text { (أطروحة دكتوراه) ، ص } 16
$$

(3) أمل وجيه حمدي مصطفى • دور المكتبة الوطنية في تحقيق التتمية المستدامة : دراسة حالة لرؤية

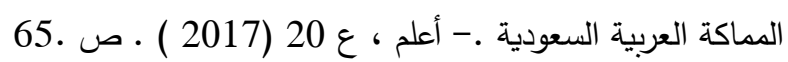

(4) أميرة محمد سيد محمود ـ رأس المال المعرفي في مكتبات جامعة القاهرة ودوره في تحقيق الميزة

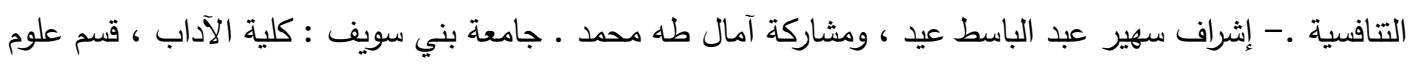

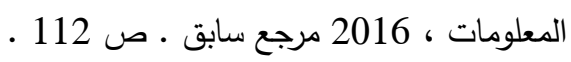

(5) إيمان محد فتحي محمود . خدمة الإمداد بالوثائق في المكتبات الوطنية :دراسة تحليلية مع وضع

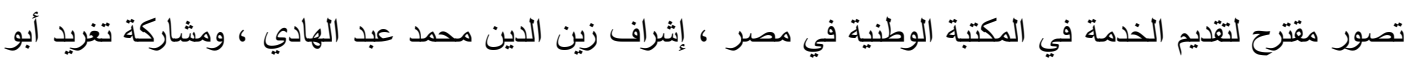

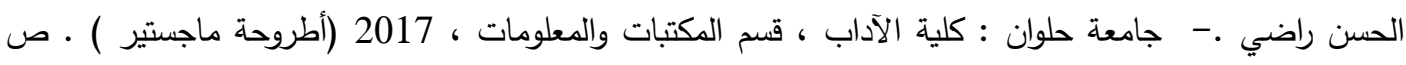
$.132-122$

(6) بدوية محمد بسيوني .الخدمة المرجعية الرقمية بالمكتبات الوطنية :دراسة تحليلية لواقعها وأساليب الإفادة

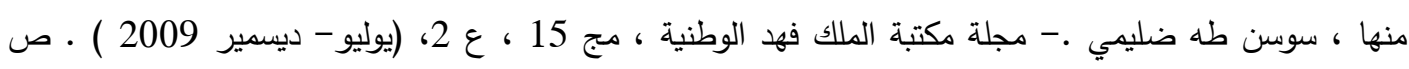
121

(7) جوزاء بنت محمد القحطاني • دور مكتبة الملك فهد الوطنية في بناء مجتمع المعرفة .- مجلة مكتبة

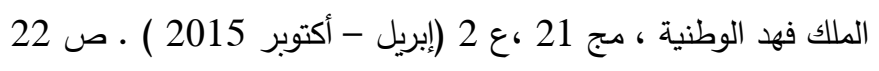

(8) حشت قاسم ـ الدكتبات الوطنية وتضافر الجهود العربية لمواجهة تحديات التراث الإكتروني .-- مجلة

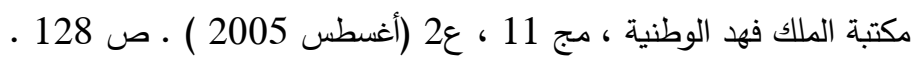

(9) خالد محمد طلال ـ تحليل معطيات العلاقة الإرتباطية بين نظام معلومات الموارد البشرية ورأس المال

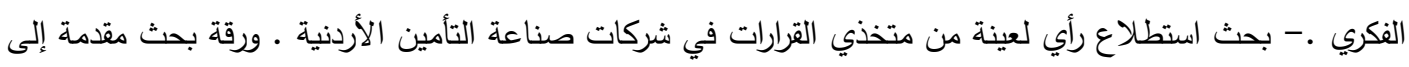


المؤتمر العلمي الأول لكلية الإقتصاد بعنوان " اقتصاد الأعمال في ظل عالم متغير . - متاح في :-210 http://abufara.net/index.php/2012-10-21-10-21-10-57 cited in 25/4/2016

(10) رحاب فايز أحمد سيد ـ قياس رأس المال المعرفي للباحثين بجامعة بني سويف : دراسة تحليلية لتحقيق الميزة التنافسية للجامعة . مجلة أعلم ، ع 16 (يناير 2016 ) . ص صدايز 24 .- متاح في : بيليhttp://www.researchgate.net/profile/Dr rehab yousef/Publications cited in 25/2/2016 علي • إقامة مجتمع المعرفة كمحر للنهضة .- المستقبل العربي، ع 342 (أغسطس 2007 ). ص ص 83- 84 . (11) رحاب فايز أحمد سيد .تقييم المكتبات الرقمية : دراسة حالة للمكتبة الرقمية العالمية .-- مجلة الإتحاد

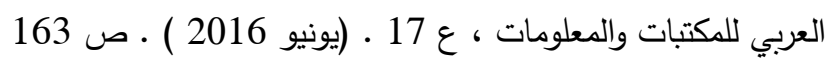

(12) زين الدين محمد عبد الهادي • رأس المال الفكري في الككتبات .- مجلة مكتبات نت ـ متاح في : http://www.ipisegypt.com/articledetails.asp?MGID=8\&vol|D=43ARID=313 cited in $15 / 5 / 2017$

السيد السيد النشار ـ أساسيات إدارة المعرفة ـ- الإسكندرية : دار الثقافة العلمية ، 2012 ـ ص 215 .

(13) سيد ربيع سيد إبراهيم .خدمات النشر العلمي على مواقع المكتبات الوطنية العربية : دراسة تطبيقية .- عابقات بحوث في علم المكتبات والمعلومات ، ع8 (مارس 2012) ـ ص 213 ـ

(14) عمر أحمد هشري . الثقافة الإلكترونية : بوابة مجتمع المعرفة ـ الحكومة والتكامل في بناء

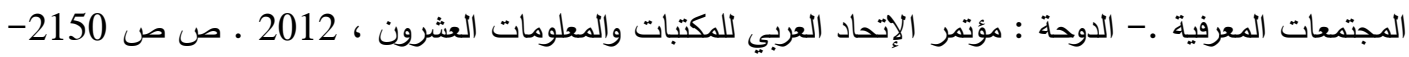

(15) محمد بن احمد نمراز حول بعض مؤشرات مجتمع المعلومات ـ- المجلة العربية للعلوم والمعلومات،

$$
\text { ع } 5 \text { (يونيو } 2005 \text { ) . ص82 }
$$

(16) محمد فتحي عبد الهادي، نبيلة خليفة جمعة ـ الككتبات الوطنية - القاهرة : الدار المصرية اللبنانية ،

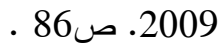

(17) محمود عبد الستار خليفة ـ الجيل الثاني من خدمات الإنترنت : مدخل إلى دراسة الويب 2 والمكتبات

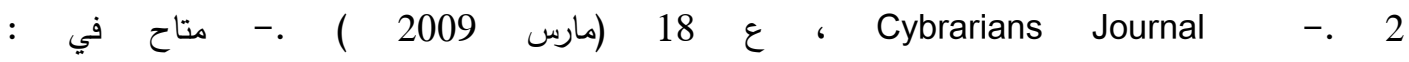
http://www.Journal.Cybrarians.org/index.php?option=com_content\&view cited in 
(18) مخلص محمد خير • المكتبات الجامعية في بيئة مجتمع المعرفة ـ إثراف زين الدين محمد عبد

الهادي ، ومشاركة إيمان فوزي عمر .- جامعة حلوان : كلية الآداب ، قسم المكتبات والمعلومات ، 2015 . أطروحة ماجستير ) · ص ص 108 . ص

(19) مي ماجد شاهين • تجارب رقمية ناجحة : تجربة مكتبة الكونجرس الأمريكية الرقمية .- مكتبات نت،

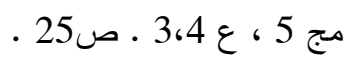

(20) هبة محمد خليفة عبد العال ـ الإعلام المكتبي الرقمي في المكتبات الوطنية : دراسة مقارنة ـ إشراف

زين الدين محمد عبد الهادي ، ومشاركة إيمان فوزي عمر .- جامعة حلوان : كلية الآداب، قسم المكتبات والمعلومات ،

$$
2015 \text { ((أطروحة ماجستير ) · ص ص } 193 \text {. }
$$

(21) يونس أحمد الشوابكة .إستخدام الفهارس العربية المتاحة للجمهور على الخط المباشر :فهرس مكتبة

$$
\begin{aligned}
& \text { الجامعة الأردنية نموذجاً.-- دراسات العلوم التربوية ،مج } 40 \text { ،ع } 1 \text { ـ ص ص 144-162. } \\
& \text { ثانياً : المراجح الأجنبة : }
\end{aligned}
$$

(1) Clair,G. Knowledge Services :The EOS International Approach.-Available at :http://www.eosintl.com/wp-content/uploads/2011/12/EOS-knowledge services (2011)

(2) Rajkumar , J . ETANA-CMV: A Coordinated Multiple view visual browsing Interface for ETANA- DL .Thesis (Msc).- Virginia Polytechnic Institute and State University . p 230 .

3)(Knowledge Services (n.d) Available at http://en.wikipedia.org/wiki/Knowledge_services cited in 3/5/2017(

(4) Koenig, Micheal . International Encyclopedia of Information and Library Science .- London.Rout- Ledge, 2003. P 351

(5) Marchionini,G and Catharine Plaisant. Interfaces and Tools for the Library of Congress National Digital Library Program .- Elsevier Science, Information Processing and Management, vol 34 (no 5) (1998). Pp 535-555

(6) Neil Beagri. National Digital preservation Initiatives : An Overview of Developments in Australia ,France,the United Kingdom and Netherlands.Washington: The Council on Library and information Resources and the Library of congress, 2003 .- Available at : http://www.clir.org/pups/reports/pup116/contents.html cited in 2/8/2016

(7) ODLIS.(n.d) .ABC-CLIO. Document Delivery Service .- Available at : http://www.abc-clio.com/odlis/searchODLIS.aspx cited in 25/6/2017 
(8) ODLIS.(n.d).ABC-CLIO. Electronic Services .- Available at : http://www.abc-clio.com/ODLIS/odlis_o.aspx\#onlineservices cited in $2 / 5 / 2017$

(9) ODLIS.(n.d).ABC-CLIO. Electronic Services .- Available at : http://www.abc-clio.com/ODLIS/odlis_o.aspx\#onlineservices cited in 2/5/2017.

(10) ODLIS.(n.d).ABC-CLIO. Electronic Services .- Available at : http://www.abc-clio.com/ODLIS/odlis_o.aspx\#onlineservices cited in 2/5/2017.

(11) RUSA. GuideLunes for Implementing and Main ting Virtual Reference Service (2014) .- Available at : http://dx.doi.org/10.5860/rusq.50nl.92 cited in $23 / 5 / 2017$.

(12) UNESCO . UNESCO National Report about ( From Information Society Towards Knowledge Society . Paris . UNESCO, 2005. - Available at : https://unesdoc.unesco.org/ark:/48223/pf0000141843_ara cited in 1/8/2015

UNESCO.Towards Information Societies .paris. UNESCO Publishing .2005.p 27.(13) ( 